\title{
RENORMALIZATION TECHNIQUES FOR QUANTUM SPIN SYSTEMS. GROUND-STATE ENERGIES
}

\section{W.J. CASPERS}

Department of Applied Physics, Twente University of Technology, Enschede, The Netherlands

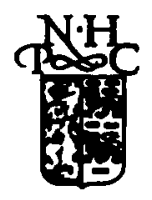

NORTH HOLLAND PUBLISHING COMPANY - AMSTERDAM 


\title{
RENORMALIZATION TECHNIQUES FOR QUANTUM SPIN SYSTEMS. GROUND-STATE ENERGIES
}

\author{
W.J. CASPERS \\ Department of Applied Physics, Twente University of Technology, Enschede, The Netherlands \\ Received January 1980
}

Contents:

1. Introduction and general outline of the method

2. Examples. Systems without external magnetic field

2.1. Linear chain with nearest and next-nearest neighbour interactions; first order

2.2. Peierls-distorted chain

2.3. Triangular lattice

2.4. Linear chain; variational method

2.5. Linear chain; second order
3. Examples. Ising and isotropic $\mathrm{XY}$ systems with a transverse magnetic field

4. Concluding remarks

Appendix A: Algebraic properties of the spin 257

Appendix B: Kramers' theorem 259

Appendix C: The Wigner-Eckart theorem 261

References $\quad 262$

Abstract:

Projection of the Hamiltonian of an antiferromagnetic lattice of spins $\frac{1}{2}$, without external fields, onto a subspace of the total spinor space gives an approximation for the lowest eigenvalue of this Hamiltonian. Repeated projection results in a series expansion for this approximation. In each projection the form of the Hamiltonian is conserved. The formal structure of this projection technique shows a strong analogy with the Wilson theory or renormalization-group theory of phase transitions. Numerical results are given for linear chains and the triangular lattice.

Analogous techniques apply to Ising and isotropic $\mathrm{XY}$ models in transverse fields.

Single orders for this issue

PHYSICS REPORTS (Review Section of Physics Letters) 63, No. 4 (1980) 223-263.

Copies of this issue may be obtained at the price given below. All orders should be sent directly to the Publisher. Orders must be accompanied by check.

Single issue price Dfl. 18.00 , postage included. 


\section{Introduction and general outline of the method}

In the last decade the Wilson theory or renormalization-group theory (RG) of phase transitions $[1,2]$ has given new possibilities for the accurate determination of critical exponents. In this paper the formal structure of the RG theory is used to determine approximate values for the lowest energy of lattices of quantum spins $\frac{1}{2}$. In most examples that will be given the approximation gives an exact upper bound. The standard formulation $[3,4,5]$ of the Wilson theory is the basis of the general outline in this section of the projection technique, which is used in this paper.

The lowest energy eigenvalue of a quantum-spin system in this work replaces the free energy in the Wilson theory (cf. Balescu [3] (10.6.2)), whereas the projection in spinor space is the parallel of the partial summation over discrete Ising variables in the partition function, used in the Wilson theory (cf. Balescu (10.6.1)). For projection as well as partial summation use is made of Kadanoff cells that replace the original constituents of the spin lattice.

Both procedures correspond with a transformation in parameter space. In the Wilson theory the equations describing this transformation are called the renormalization-group (RG) equations (cf. Balescu (10.6.4) and (10.6.5)). This name stems from quantum-field theory, which provided part of the basis of Wilson's work. The fixed points of the RG equations play an essential role in the Wilson theory, whereas in this work they only have a mathematical meaning and may sometimes be a help in evaluating numerical results.

The unlimited repetition of the RG transformation results in a series expansion for the lowest energy eigenvalue of the quantum-spin systems considered in this work.

In the first place the method is applied to antiferromagnetic lattices of spins $\frac{1}{2}$ without external magnetic field. The Kadanoff cells should always contain an odd number of spins, the lowest level of one isolated cell thus corresponding with a Kramers doublet for a dominant antiferromagnetic nearest-neighbour interaction [6]. The algebraic properties of the spin $\left(S=\frac{1}{2}\right)$ and the related Kramers' and Wigner-Eckart theorems are discussed in three appendices.

Secondly the method will be used for Ising and isotropic XY models in transverse magnetic fields.

For an outline of the method first the general spin Hamiltonian is introduced for the antiferromagnetic systems without external field

$$
H\left(S_{i} ; \gamma, \delta, \ldots\right)
$$

In this Hamiltonian the spins $S_{i}$ are situated on a $d$-dimensional lattice $(d=1,2,3, \ldots)$ with lattice vectors $i$. The interaction depends on the relative position of two spins and is characterized by a set of parameters $\gamma, \delta, \ldots$ Multiple spin interactions need not be avoided in the formalism but they are not taken into consideration in this paper. The spins always are three-dimensional Pauli spins $(D=3)$. The method is not restricted to isotropic interactions (constant $\times S_{i_{1}} \cdot S_{i_{2}}$ ), but the examples to be discussed in section 2 refer to this type only. The spin operators $S_{i}$ have components that equal $\frac{1}{2}$ times the Pauli operators, representations of which can be found in many textbooks (see e.g. ref. [7] p. 206 and appendix A).

Finite lattices of $N$ spins are considered, but final results are always calculated in the limit $N \rightarrow \infty$. Formally periodic boundary conditions are introduced in (1), but these do not play a role in the calculations. No external fields, acting on the spins, are taken into consideration in the examples of section 2. Section 3 is devoted to the ground-state energy of Ising and $X Y$ systems in transverse fields.

In order to stress the relation between formulas for the different examples in section 2 and the 
general formulas of this introduction the former are numbered $(1-1),(2-1),(2-1.1), \ldots$, the first numeral indicating the special example and the other numeral(s) the general expression in the Introduction.

The first step in the calculation for systems without external field is the introduction of Kadanoff cells $[1,2,3]$. For a linear chain, a square lattice or a cubic lattice these cells have a simple geometry: For a chain it is a collection of an odd number of consecutive spins (three in the simplest case), for a square lattice it is a square (with 9 spins in the simplest case) and for a cubic lattice a corresponding cube. For more complicated structures (cf. subsection 2.3) cells can be chosen in different ways. In every case the lattice of cells should be isomorphic with the original lattice and every spin should pertain to one and only one cell (see Niemeijer and van Leeuwen [8]).

This prescription results, for a given Kadanoff cell, in a subdivision of the original Hamiltonian (1) into two parts, $H_{0}$ and $H^{\prime}$, corresponding respectively with internal interactions within cells and interactions between cells,

$$
\begin{aligned}
& H=H_{0}+H^{\prime}, \\
& H_{0}=\sum_{\boldsymbol{k}} H_{0, \boldsymbol{k}}, \\
& H^{\prime}=\frac{1}{2} \sum_{\boldsymbol{k}} \sum_{\boldsymbol{\rho}} H_{\boldsymbol{k}, \boldsymbol{k}+\boldsymbol{\rho} .}^{\prime}
\end{aligned}
$$

The new grid $k$, indicating the centres of the cells, is isomorphic with the original one $i$. It is constructed from $i$ by scaling, which, as an example, for the linear chain with cells of 3 spins corresponds with a multiplication of the unit vector by a factor 3. The part $H_{0}$ is a sum of terms $H_{0, k}$ representing the internal interactions in cell $k$, whereas a term $H_{k, k+\rho}^{\prime}$ of $H^{\prime}$ stands for the interactions between the cells $\boldsymbol{k}$ and $\boldsymbol{k}+\boldsymbol{\rho}$.

As a next step the secular problem represented by the mutually isomorphic $H_{0, k}$ should be solved exactly. The corresponding lowest state is the direct product of a set of Kramers doublets, one per cell, at least for suitably chosen parameters $\gamma, \delta, \ldots$ of the Hamiltonian $H$, i.e. for a dominant antiferromagnetic nearest-neighbour interaction. Here the restriction to an odd number of spins per cell plays an essential role in the method. For an isotropic interaction one may choose for the lowest doublet two states with quantum numbers $S_{k}^{(1)}=\frac{1}{2}$ and $m_{k}= \pm \frac{1}{2}$ for cell $k$, the first representing the spin and the second the magnetic quantum number for a fixed $z$-direction. Also for a more general type of two-spin interaction and for suitably chosen parameters $\gamma, \delta, \ldots$ the lowest state is a Kramers doublet, which also may have the label $m_{k}= \pm \frac{1}{2}$ in the effective-spin formalism $[9,10,11,12]$. This more general case will not be worked out in this paper for the systems without external field, but the examples of section 3 make use of this formalism for non-Kramers states.

The solution of the secular problem represented by $H_{0}$, for the ground state, may be written

$$
H_{0}\left|0, m_{k}\right\rangle=\frac{N}{n} e_{0}(\gamma, \delta, \ldots)\left|0, m_{k}\right\rangle
$$

with the corresponding projection operator

$$
P_{0}=\sum_{m_{k}= \pm 1 / 2}\left|0, m_{k}\right\rangle\left\langle 0, m_{k}\right| .
$$

The quantity $e_{0}$ is the energy and $n$ the number of spins per cell. 
One could use $\boldsymbol{P}_{0}$ to project the total Hamiltonian $H$, in order to get an upper bound for its lowest eigenvalue, because the lowest eigenvalue of a projection of $H$ onto a subspace of the total spinor space always gives such an upper bound. However, a transformed projection operator $P_{1}$

$$
P_{1}=u P_{0} u^{+}
$$

$u$ being unitary, also gives an upper bound. As a consequence there is much freedom in the so-called "scaling" of the Hamiltonian $H$. This scaling is the parallel of the partial summation in the Wilson theory, at least under certain general conditions that will be formulated hereafter.

First of all the operator $u$ should have the full translational symmetry of the lattice of cells, and for the case that $H$ only contains isotropic interactions this characteristic should be preserved in the projection. This last symmetry can be guaranteed by taking $u$ operators invariant for rotations in spinor space, as is done for the example of subsection 2.4 .

Secondly the range of the interactions in the scaled lattice of cells should not exceed that of the interactions given by $H$ in the original lattice, because the aim is to produce an image of $H$, in terms of the variables corresponding with the lowest Kramers state of the cells, i.e. the spins $\boldsymbol{S}_{k}^{(\mathbf{1})}$.

Finally the value of the parameters in the Hamiltonian of the scaled problem should also correspond with a Kramers state as the lowest level for the Kadanoff cells of spins $\boldsymbol{S}_{\boldsymbol{k}}^{(1)}$. Otherwise it would not be possible to repeat the scaling, at least not with the lowest energy state of the cells. Taking an other level one would arrive at a very poor value for the upper bound of the energy.

Now the projection of $H$ will be made more explicit in order to discuss the conditions in detail. For the projection operator $P_{1}$ the projected Hamiltonian takes the form

$$
P_{1} H P_{1}=u P_{0} u^{+} H u P_{0} u^{+}
$$

which happens to have the same eigenvalues as

$$
P_{0} u^{+} H u P_{0}=H^{(1)}\left(S_{k}^{(1)} ; \gamma, \delta, \ldots\right)
$$

The last operator may be expressed in terms of the cell spins $\boldsymbol{S}_{k}^{(1)}$, because it is a projection onto the subspace of Kramers states $\left|0, m_{k}\right\rangle$. The cell-spin operators are defined by

$$
\begin{aligned}
& \left\langle 0, m_{k}^{\prime}\left|S_{i, z}^{(1)}\right| 0, m_{k}\right\rangle=\left(\prod_{k} \delta_{m_{k}, m_{k}}\right) m_{l} \\
& \left\langle 0, m_{k}^{\prime} S_{i, \pm}^{(1)} \mid 0, m_{k}\right\rangle=\left(\prod_{k \neq l} \delta_{m_{k}^{\prime}, m_{k}}\right) \delta_{m_{i, m_{I} \pm 1}} \\
& S_{l, \pm}^{(1)}=S_{i, x}^{(1)} \pm \mathrm{i} S_{i, y}^{(1)}
\end{aligned}
$$

Instead of the lowest eigenvalue of $H$ one tries to find the lowest eigenvalue of $H^{(1)}$, the latter giving an upper bound for the former. For suitably chosen $P_{0}$ and $u$ the projected Hamiltonian $H^{(1)}$ may be made isomorphic with $H$, apart from an additive and a multiplicative constant. The actual coupling constants in $H^{(1)}: \gamma^{(1)}(\gamma, \delta, \ldots), \delta^{(1)}(\gamma, \delta, \ldots), \ldots$ may have values different from $\gamma, \delta, \ldots$ To give an example in which isomorphism is not realized, one can remark that it is easy enough to generate four-spin terms in $H^{(1)}$, starting from a simple Heisenberg Hamiltonian $H$. This can be achieved by including four-spin terms in $u$. 
If isomorphism can be realized one may write

$$
H^{(1)}\left(\boldsymbol{S}_{k}^{(1)} ; \gamma, \delta, \ldots\right)=\epsilon_{0}(\gamma, \delta, \ldots) N I^{(1)}+c_{0}(\gamma, \delta, \ldots) H\left(\boldsymbol{S}_{k}^{(1)} ; \gamma^{(1)}(\gamma, \delta, \ldots), \delta^{(1)}(\gamma, \delta, \ldots), \ldots\right)
$$

The recipe that results in (5) may be called "scaling". The corresponding transformation in parameter space

$$
\begin{aligned}
& \gamma^{(1)}=\gamma^{(1)}(\gamma, \delta, \ldots) \\
& \delta^{(1)}=\delta^{(1)}(\gamma, \delta, \ldots), \ldots
\end{aligned}
$$

is the renormalization-group transformation, shortly RG transformation. The scaling is fully determined by the combination of the unitary transformation $u$ and the projection operator $\boldsymbol{P}_{0}$, both being functions, in general, of the parameters $\gamma, \delta, \ldots$ The operator $I^{(1)}$ is the identity in the space of Kramers states $\left|0, m_{k}\right\rangle$ (cf. appendix B).

If one takes into account that also for the set $\left\{\gamma^{(1)}, \delta^{(1)}, \ldots\right\}$ a Kramers state should be the lowest state for a cell there are restrictions as to the set $\{\gamma, \delta, \ldots\}$ for which the projection technique can be used. Taking for granted that there exists a region in parameter space for which the scaling may be performed an indefinite number of times it is possible to derive a series expansion for an upper bound of the lowest energy per spin. In all practical examples for which calculations have been performed, and for which scaling could be realized, this scaling results in a stable point of the RG equations. The convergence of the corresponding series for the upper bound of the energy per spin can be easily proved.

In this review three different families of unitary operators $u$ are taken into consideration. The first family simply consists of the identity operator $I$. A second family is formed by the operators $u(a, b, \ldots)$ that are functions of a set of real parameters $\{a, b, \ldots\}$. In subsection 2.4 an example is given with only one parameter. These two families, under the conditions outlined before, lead to an upper bound for the energy per spin in the ground state

$$
\begin{aligned}
& \epsilon(\gamma, \delta, \ldots) \leq \epsilon_{0}(\gamma, \delta, \ldots)+\sum_{s=1}^{\infty} \frac{1}{n^{s}}\left[\prod_{t=0}^{s-1} c_{0}\left(\gamma^{(t)}, \delta^{(t)}, \ldots\right)\right] \epsilon_{0}\left(\gamma^{(s)}, \delta^{(s)}, \ldots\right), \\
& \gamma^{(t)}=\gamma^{(1)}\left(\gamma^{(t-1)}, \delta^{(t-1)}, \ldots\right), \\
& \delta^{(t)}=\delta^{(1)}\left(\gamma^{(t-1)}, \delta^{(t-1)}, \ldots\right), \ldots, \quad t=1,2,3, \ldots, \\
& \gamma^{(0)}=\gamma, \quad \delta^{(0)}=\delta, \ldots
\end{aligned}
$$

Two remarks should be made in relation to formula (7):

(1) The functions $\gamma^{(t)}, \delta^{(t)}, \ldots$, in the case of the second family of unitary operators, also depend on the set of parameters $\left\{a^{(t-1)}, b^{(t-1)}, \ldots\right\}$ of the corresponding operator $u\left(a^{(t-1)}, b^{(t-1)}, \ldots\right)$. This results in $\mathrm{RG}$ transformations of the form:

$$
\begin{aligned}
& \gamma^{(1)}=\gamma^{(1)}(a, b, \ldots ; \gamma, \delta, \ldots), \quad \delta^{(1)}=\delta^{(1)}(a, b, \ldots ; \gamma, \delta, \ldots), \ldots \\
& \gamma^{(2)}=\gamma^{(1)}\left(a^{(1)}, b^{(1)}, \ldots ; \gamma^{(1)}, \delta^{(1)}, \ldots\right) \\
& \delta^{(2)}=\delta^{(1)}\left(a^{(1)}, b^{(1)}, \ldots ; \gamma^{(1)}, \delta^{(1)}, \ldots\right), \ldots
\end{aligned}
$$




$$
\begin{aligned}
& \gamma^{(t)}=\gamma^{(1)}\left(a^{(t-1)}, b^{(t-1)}, \ldots ; \gamma^{(t-1)}, \delta^{(t-1)}, \ldots\right), \\
& \delta^{(t)}=\delta^{(1)}\left(a^{(t-1)}, b^{(t-1)}, \ldots ; \gamma^{(t-1)}, \delta^{(t-1)}, \ldots\right), \ldots \\
& \cdots \ldots \ldots \ldots \\
& a^{(0)}=a, \quad b^{(0)}=b, \ldots
\end{aligned}
$$

In (7) the minimum value for all possible sets of parameters $\left\{a^{(t-1)}, b^{(t-1)}, \ldots\right\}, t=1,2,3, \ldots$ is chosen to give the best, i.e. the lowest upper bound for $\epsilon$.

(2) Repeated scaling according to (5) gives a rest term in operator form. For the set $\left\{\gamma^{(t)}, \delta^{(t)}, \ldots\right\}$ converging to a stable point it is easy to show that this rest term gives a vanishing contribution to the upper bound for $\epsilon$, in the case: $t \rightarrow \infty$.

As a third family of unitary operators one may take those $u$ that are given by the RayleighSchrödinger perturbation theory in second order. These operators, however, are not exactly unitary and the corresponding series expansion no longer gives an upper bound, because the corresponding $u P_{0} u^{+}$ no longer is an exact projection operator. The series expansion (7) should be replaced by one of the same general form, giving an approximated value for the energy per spin in the ground state:

$$
\epsilon(\gamma, \delta, \ldots) \approx \epsilon_{0}(\gamma, \delta, \ldots)+\sum_{s=1}^{\infty} \frac{1}{n^{s}}\left[\prod_{t=0}^{s-1} c_{0}\left(\gamma^{(t)}, \delta^{(t)}, \ldots\right)\right] \epsilon_{0}\left(\gamma^{(s)}, \delta^{(s)}, \ldots\right)
$$

In section 2 five examples are discussed in detail. The linear chain with nearest and next-nearest neighbour interactions in subsection 2.1, the Peierls-distorted chain (subsection 2.2) and the triangular lattice (subsection 2.3) are examples for which $u=I$. In subsection 2.4 a variational operator $u=u(a)$ with one (real) parameter is used for the linear chain, as an example of the second family. Finally in 2.5 the linear chain is treated again, here with second-order perturbation theory. The approximated value for the energy per spin in the ground state will then be given by a series of the type (8).

For every example a comparison is made with other values for $\epsilon$ in the literature. Part of this discussion, in particular for the linear chain, is postponed till section 4.

Analogous methods for the determination of the ground-state energy of quantum-spin systems have been developed recently by Fields et al. [13] and Dekeyser [14].

In section 3 the work of Jullien, Pfeuty, Fields, Doniach and Penson on Ising and XY models in transverse fields is discussed $[15,16,17]$. It shows a strong analogy with the work on spin systems without external fields, but in the RG transformation one extra parameter has to be taken into consideration, i.e. the external magnetic field the value of which is also changed in the scaling operation. In this review no attention is paid to any effects at temperatures $T>0$.

\section{Examples. Systems without external magnetic field}

The examples given in this section have already been analyzed in a series of papers [18]-[22] by the author in co-operation with many others. Recent work of other groups has been cited in the Introduction $[13,14]$. Here the examples are shown as applications of the general formalism developed in section 1. 
2.1. Linear chain with nearest and next-nearest neighbour interactions; first order (van de Braak, Caspers, de Lange and Willemse [18])

The Hamiltonian (1) of the Introduction now takes the form

$$
H\left(S_{i} ; \gamma\right)=4 \sum_{i}\left(S_{i} \cdot S_{i+1}+\gamma S_{i} \cdot S_{i+2}\right)
$$

The energy has been normalized in such a way that the coupling constant for neighbour spins equals 4 . The only variable parameter $\gamma$ measures the ratio of the strengths of the next-nearest neighbour and of the nearest neighbour interactions. Both are scalar interactions of the well-known Heisenberg type:

The proper Kadanoff cell for this system is an odd number of consecutive spins, and the simplest example of such a cell contains three spins. For such cells the subdivision of the Hamiltonian according to (2) takes the form

$$
\begin{aligned}
& H=H_{0}+H^{\prime}, \\
& H_{0}=\sum_{k} H_{0, k}, \quad H_{0, k}=4\left(S_{3 k-1} \cdot S_{3 k}+S_{3 k} \cdot S_{3 k+1}\right)+4 \gamma S_{3 k-1} \cdot S_{3 k+1}, \\
& H^{\prime}=\sum_{k} H_{k, k+1}^{\prime}, \quad H_{k, k+1}^{\prime}=4 S_{3 k+1} \cdot S_{3 k+2}+4 \gamma\left(S_{3 k} \cdot S_{3 k+2}+S_{3 k+1} \cdot S_{3 k+3}\right) .
\end{aligned}
$$

The cell index $k$ represents points of a grid with a unit distance that is the triple of the original unit.

For an isolated cell the secular problem, represented by one single $H_{0, k}$, is easily solved. For $\gamma<1$ the ground state is a Kramers doublet $\left|\frac{1}{2}+, m_{k}\right\rangle, m_{k}= \pm \frac{1}{2}$, the spin quantum number $S_{k}$ being $\frac{1}{2}$. The + sign indicates the symmetry character for interchanging the spins at the sites $3 k-1$ and $3 k+1$. The excited states are an antisymmetric doublet $\left|\frac{1}{2}-, m_{k}\right\rangle$ and a (symmetric) triplet $\left|\frac{3}{2}, m_{k}\right\rangle$, which are given in subsection 2.5 .

Omitting the cell index $k$, one may write the symmetric doublet as

$$
\begin{aligned}
& \left|\frac{1}{2}+, \frac{1}{2}\right\rangle=-\frac{1}{\sqrt{6}}[2|+-+\rangle-(|-++\rangle+|++-\rangle)], \\
& \left|\frac{1}{2}+,-\frac{1}{2}\right\rangle=\frac{1}{\sqrt{6}}[2|-+-\rangle-(|+--\rangle+|--+\rangle)],
\end{aligned}
$$

in which, as an example, the ket $|+-+\rangle$ indicates a spinor corresponding with a magnetic quantum number $+\frac{1}{2}$ for the spins at positions $3 k \pm 1$ and $-\frac{1}{2}$ for the spin at position $3 k$.

The eigenvalue of $H_{0, k}$ corresponding with this Kramers doublet is

$$
e_{0}(\gamma)=-4+\gamma
$$

and the solution of the unperturbed secular problem represented by $H_{0}$ is

$$
H_{0}\left|0, m_{k}\right\rangle=\frac{N}{3} e_{0}(\gamma)\left|0, m_{k}\right\rangle, \quad\left|0, m_{k}\right\rangle=\prod_{k}\left|\frac{1}{2}+, m_{k}\right\rangle
$$


The $N$ spins of the linear system are supposed to be arranged in a closed ring, which realizes the proper periodic boundary condition.

Now the projected Hamiltonian will be calculated. It is represented by

$$
P_{0} H P_{0}(u=I), \quad P_{0}=\sum_{m_{k}}\left|0, m_{k}\right\rangle\left\langle 0, m_{k}\right|
$$

In the projection the internal part $H_{0}$ of the Hamiltonian simply gives $(N / 3) e_{0}(\gamma) I^{(1)}$, whereas for the determination of $P_{0} H^{\prime} P_{0}$ one needs the projections of the spin vectors $S_{3 k}, S_{3 k \pm 1}$. These projections can be expressed in terms of the vector $\boldsymbol{S}_{k}^{(1)}$, according to the Wigner-Eckart theorem, which is discussed in appendix $\mathrm{C}$. The reader is also referred to a monograph by Edmonds [23].

For practical calculations one only needs to calculate one matrix element, e.g.

$$
\left\langle\frac{1}{2}+, m_{k}^{\prime}=\frac{1}{2}\left|\boldsymbol{S}_{3 k, z}\right| \frac{1}{2}+, m_{k}=\frac{1}{2}\right\rangle,
$$

from which all others may be derived taking into account the symmetry of the positions $3 k-1$ and $3 k+1$ and the identity: $\boldsymbol{P}_{0}\left(\boldsymbol{S}_{3 k-1}+\boldsymbol{S}_{3 k}+\boldsymbol{S}_{3 k+1}\right) \boldsymbol{P}_{0}=\boldsymbol{S}_{k}^{(1)}$. For the cell-spin operators $\boldsymbol{S}_{k}^{(1)}$ general definitions are given in the Introduction, in particular formula (4).

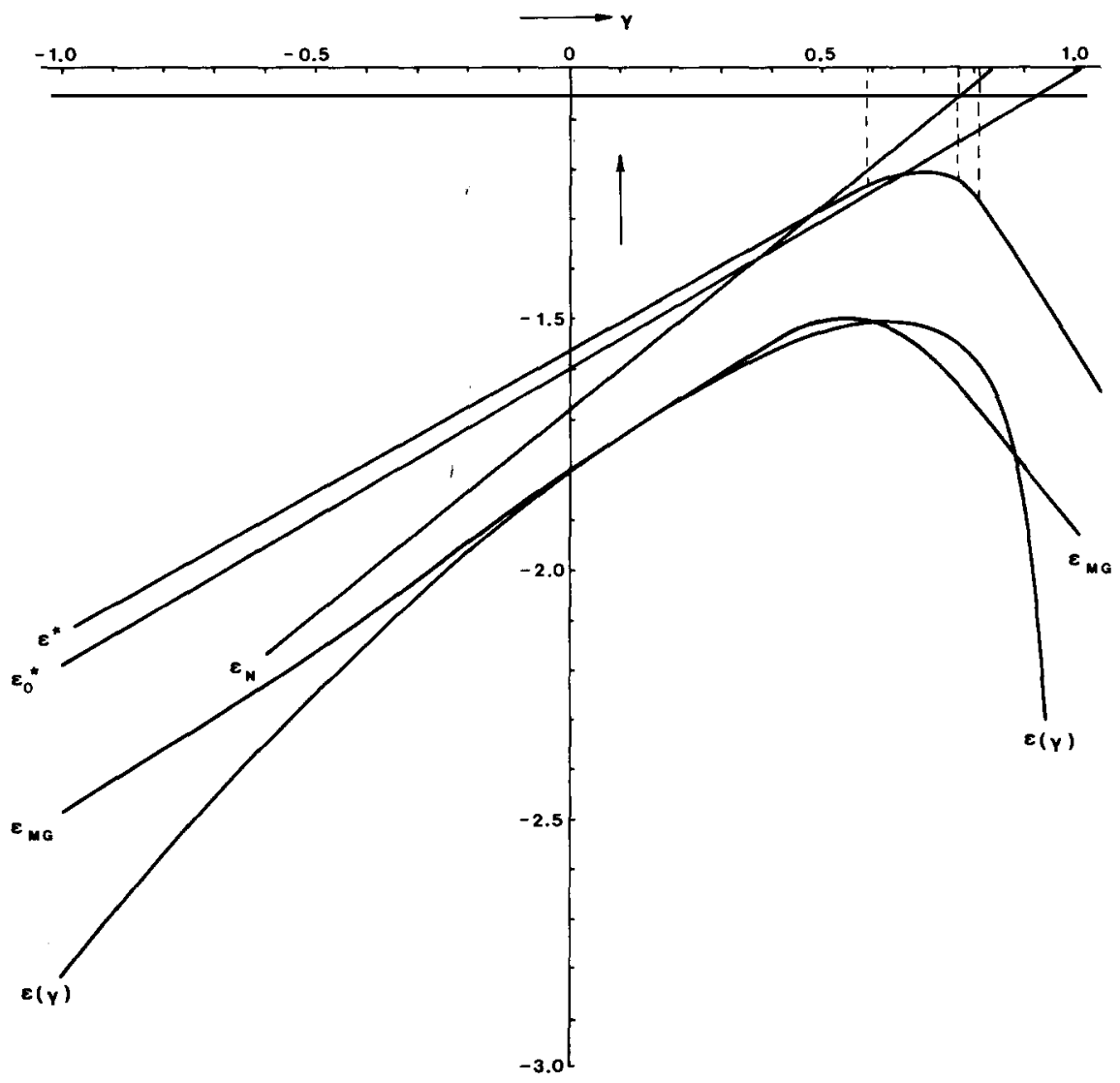

Fig. 1. Energy per spin for the linear chain $[18,21,22]$. 
So one finds for the projected single spin operators

$$
P_{0} S_{3 k} P_{0}=-\frac{1}{3} S_{k}^{(1)}, \quad P_{0} S_{3 k \pm 1} P_{0}=\frac{2}{3} S_{k}^{(1)}
$$

and the projected Hamiltonian (5), for the example on hand, takes the form

$$
\begin{aligned}
& H^{(1)}\left(S_{k}^{(1)} ; \gamma\right)=\frac{1}{3}(-4+\gamma) N I^{(1)}+\frac{16}{9}(1-\gamma) \sum_{k} S_{k}^{(1)} \cdot S_{k+1}^{(1)}, \\
& \epsilon_{0}(\gamma)=\frac{1}{3}(-4+\gamma)=\frac{1}{3} e_{0}(\gamma) \\
& c_{0}(\gamma)=\frac{4}{9}(1-\gamma) .
\end{aligned}
$$

The transformation in parameter space or $\mathrm{RG}$ transformation is trivial,

$$
\gamma^{(1)}(\gamma)=0
$$

and the scaled secular problem (1-5) is exactly solvable, because $H^{(1)}$ is the Hamiltonian of a Heisenberg chain, with only nearest-neighbour interactions, apart from an additive constant. In this example the upper bound for $\epsilon$ is not given as an infinite series but as a closed expression.

Making use of Hulthén's exact result [24] one finds the upper bound $\epsilon_{0}^{*}(\gamma)$ for the energy per spin

$$
\epsilon(\gamma) \leq \epsilon_{0}^{*}(\gamma)=\frac{1}{3}(-4+\gamma)+\frac{4}{9}(1-\gamma) \frac{1}{3}(1-4 \log 2)=-1.5960+0.5960 \gamma
$$

The curve for $\epsilon_{0}^{*}(\gamma)$ is drawn in fig. 1, which also gives the other approximations of the subsections 2.4 and 2.5, together with other results in the literature. Detailed comparison between the different values is postponed till section 4 .

\subsection{Peierls-distorted chain (van de Braak, Caspers, Wiegel and Willemse [19])}

In a spin chain with the possibility of elastic deformation and a spin coupling that depends on the mutual displacements, spontaneous deformation, in particular dimerization, may occur [25]-[32]. Here only the dimerized or Peierls-distorted chain is taken into consideration. In the Peierls-distorted linear chain of $N$ spins there is an alternating coupling constant for nearest-neighbour pairs, depending on a distortion parameter $\xi$. The corresponding Hamiltonian (1), in which next-nearest-neighbour interactions etc. are not taken into account, has the form

$$
H\left(S_{i} ; \xi\right)=-J_{0} \sum_{i}\left(1+(-)^{i} \xi\right) S_{i} \cdot S_{i+1}, \quad J_{0}<0
$$

The distortion results in a classical elastic energy of the lattice

$$
E_{l}(\Delta)=N \omega_{0} \Delta^{2}, \quad \xi=\gamma \Delta
$$

the kinetic energy of the lattice not being taken into consideration in the evaluation of the energy of the ground state of the total system. In formula (2-1.1) the parameter $\Delta$ is the displacement of the lattice points in an absolute sense, the relative change of the coupling constants in (2-1) being a linear function of this parameter. The lattice distortion has a wavelength of two elementary distances and results in a 
doubling of the cell: consecutive lattice points are shifted in opposite directions. The spin interaction again is of the well-known isotropic Heisenberg type.

Peierls distortion has been analyzed in a number of papers by Pincus [25], Beni and Pincus [26], Dubois and Carton [27], Beni [28], Pytte [29], Jacobs et al. [30], Penson et al. [31] and Cross and Fisher [32]. In this subsection the notation of ref. [31] is used. Quite independently from the author RG methods have been applied to dimerized chains by Fields, Blöte and Bonner [13]. For Kadanoff cells of three spins the subdivision of the Hamiltonian for this system may be written as

$$
\begin{aligned}
& H=H_{0}+H^{\prime}, \\
& H_{0}=\sum_{k} H_{0, k}, \quad H_{0, k}=-J_{0}\left[\left(1-(-)^{k} \xi\right) S_{3 k-1} \cdot S_{3 k}+\left(1+(-)^{k} \xi\right) S_{3 k} \cdot S_{3 k+1}\right], \\
& H^{\prime}=\sum_{k} H_{k, k+1}^{\prime}, \quad H_{k, k+1}^{\prime}=-J_{0}\left(1-(-)^{k} \xi\right) S_{3 k+1} \cdot S_{3 k+2} .
\end{aligned}
$$

The eigenvalues of $H_{0, k}$ correspond with one quartet (cell spin $S_{k}^{(1)}=\frac{3}{2}$ ) and two doublets (cell spin $\left.S_{k}^{(1)}=\frac{1}{2}\right)$ :

$$
\begin{array}{ll}
-\frac{1}{2} J_{0} & \left(S_{k}^{(1)}=\frac{3}{2}\right), \\
\frac{1}{2} J_{0}\left(1 \pm\left(1+3 \xi^{2}\right)^{1 / 2}\right) & \left(S_{k}^{(1)}=\frac{1}{2}\right) .
\end{array}
$$

For all (real) $\xi$ the lowest level is the doublet with energy $\frac{1}{2} J_{0}\left(1+\left(1+3 \xi^{2}\right)^{1 / 2}\right)$ because $J_{0}<0$. The corresponding eigenstates are

$$
\begin{aligned}
& \left|\frac{1}{2}, \pm \frac{1}{2}\right\rangle_{k}=\frac{1}{\sqrt{6}}\left[\mp 2 \alpha_{k}| \pm \mp \pm\rangle_{k} \pm\left(\alpha_{k}+\sqrt{3} \beta_{k}\right)|\mp \pm \pm\rangle_{k} \pm\left(\alpha_{k}-\sqrt{3} \beta_{k}\right)| \pm \pm \mp\rangle_{k}\right] \\
& \alpha_{k}=\frac{1}{\sqrt{2}} \frac{\eta_{k}}{\left(1+\eta_{k}^{2}-\left(1+\eta_{k}^{2}\right)^{1 / 2}\right)^{1 / 2}} \\
& \beta_{k}=\frac{1}{\sqrt{2}} \frac{1-\left(1+\eta_{k}^{2}\right)^{1 / 2}}{\left(1+\eta_{k}^{2}-\left(1+\eta_{k}^{2}\right)^{1 / 2}\right)^{1 / 2}}, \quad \eta_{k}=(-)^{k} \xi \sqrt{3}
\end{aligned}
$$

The kets $|+-+\rangle_{k}$ etc. with a subscript referring to the $k$ th cell have the same definition as in the Introduction. So the internal energy of a cell in its ground state,

$$
e_{0}(\xi)=\frac{1}{2} J_{0}\left(1+\left(1+3 \xi^{2}\right)^{1 / 2}\right)
$$

is independent of the cell index $k$ and the solution of the secular problem represented by $H_{0}$ is

$$
H_{0}\left|0, m_{k}\right\rangle=\frac{N}{3} e_{0}(\xi)\left|0, m_{k}\right\rangle, \quad\left|0, m_{k}\right\rangle=\prod_{k}\left|\frac{1}{2}, m_{k}\right\rangle_{k}
$$

For the scaling of the Hamiltonian in this example again the projection operator $P_{0}$,

$$
P_{0}=\sum_{m_{k}= \pm 1 / 2}\left|0, m_{k}\right\rangle\left\langle 0, m_{k}\right|
$$

will be used, corresponding with the trivial unitary transformation, $u=I$. 
The projected spin operators, written in terms of cell spins, are

$$
P_{0} S_{3 k} P_{0}=\frac{1}{3}\left(-\alpha_{k}^{2}+3 \beta_{k}^{2}\right) S_{k}^{(1)}, \quad P_{0} S_{3 k \pm 1} P_{0}=\frac{2}{3} \alpha_{k}\left(\alpha_{k} \pm \sqrt{3} \beta_{k}\right) S_{k}^{(1)}
$$

and the projected Hamiltonian reads

$$
\begin{aligned}
& H^{(1)}\left(S_{k}^{(1)} ; \xi\right)=\epsilon_{0}(\xi) N I^{(1)}+c_{0}(\xi) H\left(S_{k}^{(1)} ; \xi^{(1)}(\xi)\right) \\
& \epsilon_{0}(\xi)=\frac{1}{6} J_{0}\left(1+\left(1+3 \xi^{2}\right)^{1 / 2}\right)=\frac{1}{3} e_{0}(\xi) \\
& c_{0}(\xi)=\frac{2 \xi^{2}}{\left[1+3 \xi^{2}-\left(1+3 \xi^{2}\right)^{1 / 2}\right]^{2}}\left[1+\xi^{2}-\left(1-\xi^{2}\right)\left(1+3 \xi^{2}\right)^{1 / 2}\right]
\end{aligned}
$$

with a transformed distortion parameter $\xi^{(1)}(\xi)$

$$
\xi^{(1)}(\xi)=\Gamma(\xi)=-\frac{2 \xi^{3}}{1+\xi^{2}-\left(1-\xi^{2}\right)\left(1+3 \xi^{2}\right)^{1 / 2}}
$$

Careful inspection shows that projection indeed results in proper scaling with the correct symmetry of $H^{(1)}$ : The distortion for adjacent pairs of cell spins has opposite sign as a consequence of the number of spins per cell being odd.

Again the scaling procedure leading to $H^{(1)}$ may be repeated indefinitely, yielding an upper bound for the ground-state energy per spin

$$
\begin{aligned}
& \epsilon(\xi) \leq \epsilon^{*}(\xi)=\sum_{s=0}^{\infty} \frac{1}{3^{s}}\left(\prod_{t=0}^{s-1} c_{0}\left(\xi^{(t)}\right)\right) \epsilon_{0}\left(\xi^{(s)}\right) \\
& \xi^{(s)}=\Gamma\left(\xi^{(s-1)}\right), \quad \prod_{t=0}^{-1} c_{0}\left(\xi^{(t)}\right)=1 \\
& \xi^{(0)}=\xi
\end{aligned}
$$

The actual Peierls distortion is determined by the minimum of the total energy per spin, including the elastic energy (2-1.1), i.e. the minimum of

$$
w(\xi)=\epsilon^{*}(\xi)+\frac{E_{l}(\Delta)}{N}=\epsilon^{*}(\xi)+\frac{\omega_{0}}{\gamma^{2}} \xi^{2}
$$

Numerical results [19] of the renormalization method for the Peierls-distorted chain are shown in four graphs and three tables. First of all fig. 2 shows the graph of $\Gamma(\xi)$, the function that determines the RG transformation. Restriction is made to the interval $[0,1.5]$ for $\xi$, because $\Gamma(\xi)$ is an odd function. Asymptotic values of $\Gamma(\xi)$ are

$$
\begin{aligned}
& \Gamma(\xi) \sim-4 \xi(\xi \rightarrow 0), \\
& \Gamma(\xi) \sim-2 / \sqrt{ } 3=-1.1547(\xi \rightarrow \infty) .
\end{aligned}
$$




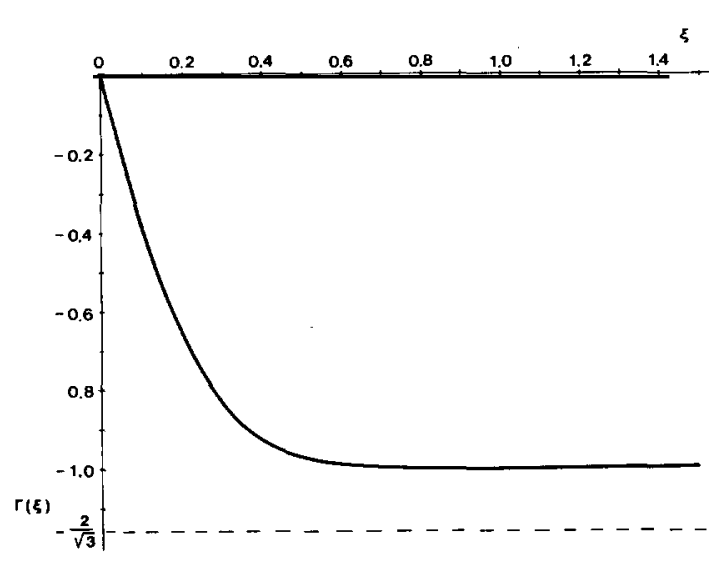

Fig. 2. The function $\Gamma(\xi)[19]$.

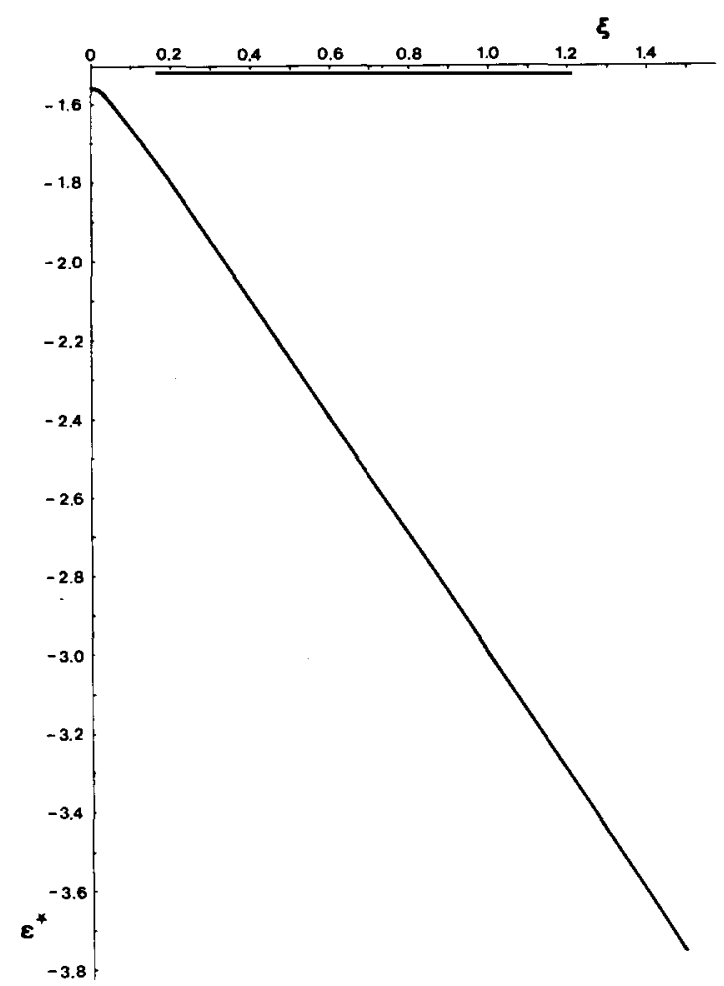

Fig. 3. The ground-state energy per spin $\epsilon^{*}(\xi)$ [19].

The renormalization gives a change of sign of the distortion parameter. Fixed points of the RG transformation are given by the equation $\Gamma(\xi)=-\xi$, which for $\xi \geq 0$ has the solutions $\xi=0,1$. The stable point $\left|\xi^{(s)}\right|=1$ is reached, with sufficient accuracy, after a reasonable number of transformations, for all values $\xi^{(0)}=\xi$ in the interval $[0,1.5]$. The values of $\epsilon^{*}(\xi)$, given by (2-7), are shown in fig. 3 and in table 1 , for a coupling constant $J_{0}=-4$.

For the fixed points one finds the values $\epsilon^{*}(0)=-\frac{36}{23}=-1.5652$ and $\epsilon^{*}(1)=-3$. For the calculation of table 1 and the other numerical results of this subsection use has been made of a Wang 500 desk calculator.

The function $\epsilon^{*}(\xi)$ is monotonic for $\xi>0$. Its behaviour for $\xi \geq 0$ is determined by a characteristic exponent $\alpha$, which is calculated in the following lines.

Table 1

$\epsilon^{*}(\xi)$ as a function of $\xi[19]$

\begin{tabular}{ll|ll}
\multicolumn{4}{c|}{$\epsilon^{*}(\xi)$ as a function of $\xi[19]$} \\
\hline$\xi$ & $\epsilon^{*}(\xi)$ & $\xi$ & $\epsilon^{*}(\xi)$ \\
\hline 0 & $-1.565217\left(=-\frac{36}{23}\right)$ & 0.8 & -2.699697 \\
0.1 & -1.659742 & 0.9 & -2.849966 \\
0.2 & -1.792566 & 1.0 & -3 \\
0.3 & -1.938978 & 1.1 & -3.150028 \\
0.4 & -2.091136 & 1.2 & -3.300204 \\
0.5 & -2.244506 & 1.3 & -3.450625 \\
0.6 & -2.397225 & 1.4 & -3.601343 \\
0.7 & -2.548894 & 1.5 & -3.752386 \\
\hline
\end{tabular}


First three new functions are introduced (cf. (2-5))

$$
\begin{aligned}
& \bar{\epsilon}^{*}(\xi)=\epsilon^{*}(\xi)-\epsilon^{*}(0)=\epsilon^{*}(\xi)+\frac{36}{23} \\
& \bar{c}_{0}(\xi)=c_{0}(\xi)-c_{0}(0)=c_{0}(\xi)-\frac{4}{9} \\
& \bar{\epsilon}_{0}(\xi)=\epsilon_{0}(\xi)-\epsilon_{0}(0)=\epsilon_{0}(\xi)+\frac{4}{3}
\end{aligned}
$$

The function $\epsilon^{*}(\xi)$, according to (2-7), obeys the relation

$$
\epsilon^{*}(\xi)-\epsilon_{0}(\xi)=\frac{1}{3} c_{0}(\xi) \epsilon^{*}\left(\xi^{(1)}\right), \quad \xi^{(1)}=\Gamma(\xi),
$$

which in terms of the new functions $\bar{\epsilon}^{*}, \bar{c}_{0}$ and $\bar{\epsilon}_{0}$ reads

$$
\bar{\epsilon}^{*}(\xi)-\bar{\epsilon}_{0}(\xi)=-\frac{12}{23} \bar{c}_{0}(\xi)+\left(\frac{4}{27}+\frac{1}{3} \bar{c}_{0}(\xi)\right) \bar{\epsilon}^{*}\left(\xi^{(1)}\right) .
$$

Introducing the characteristic exponent $\alpha$ by $\bar{\epsilon}^{*}(\xi) \simeq k_{1}|\xi|^{\alpha}(|\xi| \rightarrow 0)$, one easily determines the dominant terms in (2-7.2) for small $|\xi|$. Doing so one finds

$$
k_{1}|\xi|^{\alpha}=\frac{4}{27} k_{1} 4^{\alpha}|\xi|^{\alpha}, \quad \alpha=\frac{3 \ln 3}{2 \ln 2}-1=1.37744
$$

Because $\alpha<2$ the term $\bar{\epsilon}_{0}(\xi)$ of the left-hand side and the terms corresponding with $\bar{c}_{0}(\xi)$ of the right-hand side of (2-7.2) do not contribute to the dominant part. The value of $\alpha$, found in this way, is confirmed by the numerical results given in table 1 .

For large values of $\xi, \xi^{(1)}=\Gamma(\xi) \simeq-2 / \sqrt{3}, c_{0}(\xi) \simeq \frac{2}{9} \xi \sqrt{3}$ and $\epsilon_{0}(\xi) \simeq-\frac{2}{3} \sqrt{3} \xi$, thus

$$
\epsilon^{*}(\xi)=\epsilon_{0}(\xi)+\frac{1}{3} c_{0}(\xi) \epsilon^{*}\left(\xi^{(1)}\right) \simeq\left[-\frac{2}{3} \sqrt{3}+\frac{1}{3} \cdot \frac{2}{9} \sqrt{3} \epsilon^{*}(-2 / \sqrt{3})\right] \xi=-1.56960 \xi
$$

The minimum value of the function $w(\xi)$ given by (2-7.1) is easily determined numerically from the results for $\epsilon^{*}(\xi)$. The value $\xi_{0}$ of $\xi$, for which this minimum is reached, as a function of $\omega_{0} / \gamma^{2}$, is given in fig. 4 and table 2.

The final graph in fig. 5 is that of $w\left(\xi_{0}\right)$, the minimal energy of the distorted chain, as a function of the elastic constant $\omega_{0} / \gamma^{2}$. Table 3 gives the corresponding numerical values, together with those for $\epsilon^{*}\left(\xi_{0}\right)$.

In the approximation for the ground state of the Peierls chain given here, there is a finite distortion for all non-zero values of $\omega_{0} / \gamma^{2}$, as a consequence of the characteristic exponent $\alpha$ being smaller than 2, consequently $\partial w / \partial \xi<0$ for $\xi=0$.

In the normalization of this paper Hulthén's result [24] equals $\epsilon_{\mathrm{H}}(0)=1-4 \ln 2=-1.7726$. The renormalization method gives $w\left(\xi_{0}\right)<\epsilon_{\mathrm{H}}(0)$ for $\omega_{0} / \gamma^{2}<1.994$, so that for these values of $\omega_{0} / \gamma^{2}$ there is a strong indication that Peierls distortion occurs for the exact ground state, because the value of $w\left(\xi_{0}\right)$, being an upper bound for the exact energy, is smaller than Hulthén's result. This last result corresponds with an undistorted chain.

For the rigid lattice $\left(\omega_{0} \rightarrow \infty\right)$ the method of this subsection may be easily improved. Here one finds $\xi_{0}=0$ and the corresponding value of $\epsilon^{*}$ equals: $-36 / 23=-1.5652$, whereas, even in the rather simple approximation of subsection 2.1 a better value is found: $\epsilon_{0}^{*}(0)=-1.5960$, still showing a discrepancy of 


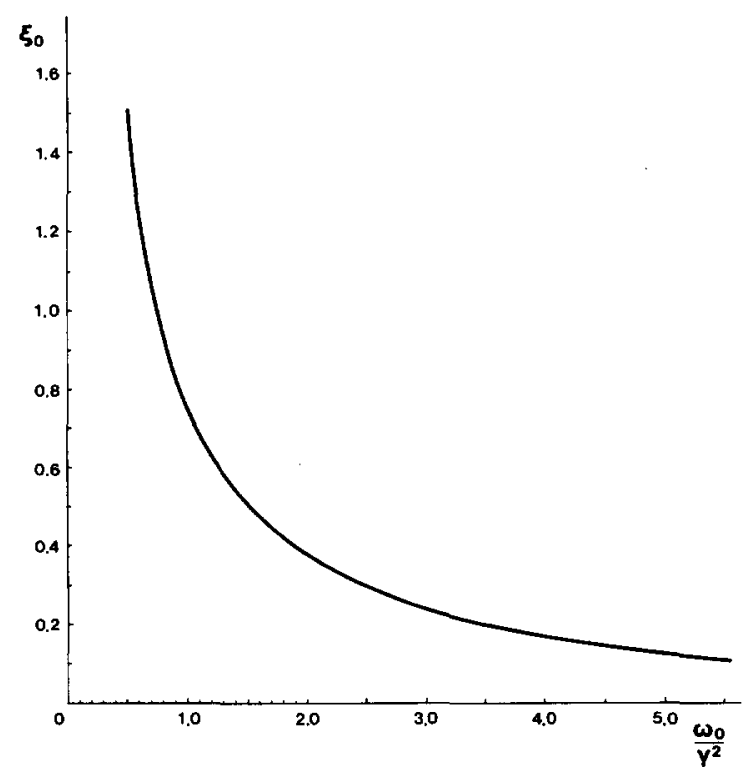

Fig. 4. The distortion parameter $\xi_{0}$ for minimum energy, as a function of $\omega_{0} / \gamma^{2}[19]$.

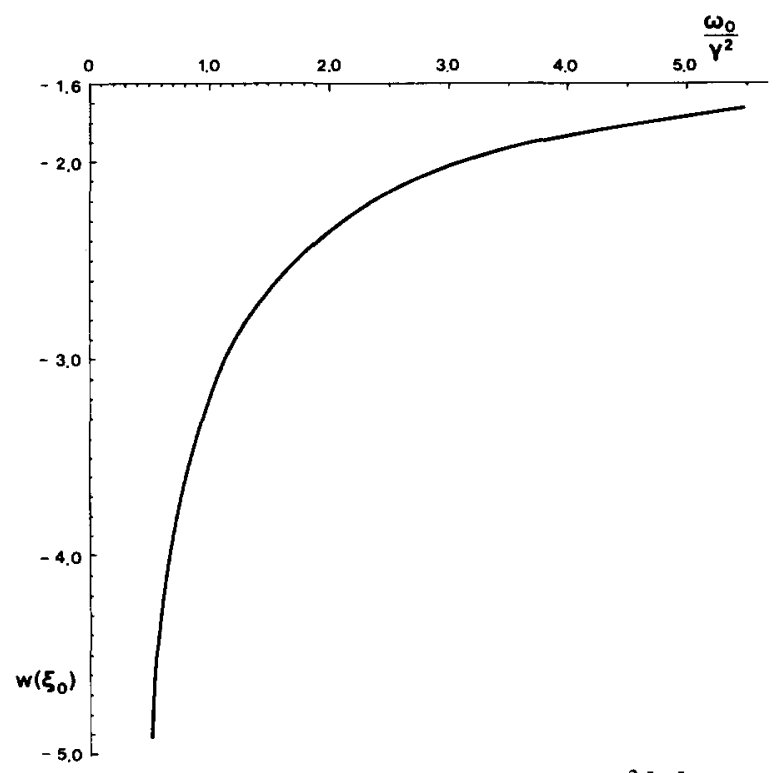

Fig. 5. The minimal energy $w\left(\xi_{0}\right)$ as a function of $\omega_{0} / \gamma^{2}[19]$.

$11 \%$ with the exact value of Hulthén. The variational method of subsection 2.4 gives the same value as this subsection, whereas in 2.5 an overestimate of the absolute value of $\epsilon(0)$ is found with second-order perturbation calculus.

The renormalization results for $\xi_{0}$, given in fig. 4 and table 2 may be compared with those of Pincus [25] and Beni [28]. In the first paper the analogous problem for the XY model is solved exactly, resulting in $\xi_{0} \approx \exp \left(-\pi \omega_{0} / 4 \gamma^{2}\right)\left(\xi_{0} \ll 1\right)$, expressed in terms of the corresponding constants in the analysis of the present work. For the Heisenberg interaction in the Hartree-Fock approximation it was found in ref. [28] that $\xi_{0} \approx \exp (-\bar{a})\left(\xi_{0} \ll 1\right)$, where $\bar{a}=2 a /\left[1+(1+8 a / \pi)^{1 / 2}\right]$ and $a=\pi \omega_{0} / 4 \gamma^{2}$.

Pytte [29] has analyzed the Peierls distortion for a linear antiferromagnetic chain in interaction with three-dimensional phonons. Jacobs et al. [30] have given a survey of the energy lowering of the ground state near the uniform limit $(\xi=0)$ : For the $X Y$ model it goes like $\xi^{2} \ln \xi[25,27]$ and for the

Table 2

$\xi_{0}$ as a function of $\omega_{0} / \gamma^{2}[19]$

\begin{tabular}{ll|ll}
\hline$\omega_{0} / \gamma^{2}$ & $\xi_{0}$ & $\omega_{0} / \gamma^{2}$ & $\xi_{0}$ \\
\hline 0.5 & 1.5125 & 1.5 & 0.5104 \\
0.6 & 1.2536 & 2.0 & 0.3826 \\
0.7 & 1.0714 & 2.5 & 0.3007 \\
0.8 & 0.9377 & 3.0 & 0.2436 \\
0.9 & 0.8351 & 3.5 & 0.2021 \\
1.0 & 0.7538 & 4.0 & 0.1713 \\
1.1 & 0.6877 & 4.5 & 0.1477 \\
1.2 & 0.6327 & 5.0 & 0.1293 \\
1.3 & 0.5859 & 5.5 & 0.1145 \\
1.4 & 0.5456 & & \\
\hline
\end{tabular}

Table 3

$\omega\left(\xi_{0}\right)$ and $\epsilon^{*}\left(\xi_{0}\right)$ as a function of $\omega_{0} / \gamma^{2}[19]$

\begin{tabular}{lll|lll}
\hline$\omega_{0} / \gamma^{2}$ & $w\left(\xi_{0}\right)$ & $\epsilon^{*}\left(\xi_{0}\right)$ & $\omega_{0} / \gamma^{2}$ & $w\left(\xi_{0}\right)$ & $\epsilon^{*}\left(\xi_{0}\right)$ \\
\hline 0.5 & -2.6275 & -3.7713 & 1.5 & -1.8697 & -2.2604 \\
0.6 & -2.4379 & -3.3807 & 2.0 & -1.7717 & -2.0645 \\
0.7 & -2.3036 & -3.1071 & 2.5 & -1.7140 & -1.9400 \\
0.8 & -2.2031 & -2.9066 & 3.0 & -1.6772 & -1.8552 \\
0.9 & -2.1248 & -2.7525 & 3.5 & -1.6526 & -1.7956 \\
1.0 & -2.0619 & -2.6301 & 4.0 & -1.6352 & -1.7526 \\
1.1 & -2.0101 & -2.5303 & 4.5 & -1.6226 & -1.7208 \\
1.2 & -1.9666 & -2.4469 & 5.0 & -1.6130 & -1.6966 \\
1.3 & -1.9295 & -2.3758 & 5.5 & -1.6056 & -1.6777 \\
1.4 & -1.8975 & -2.3143 & & & \\
\hline
\end{tabular}


Heisenberg model in the HF approximation like $\xi^{2}(\ln \xi)^{2}[28,29]$, whereas in the first-order approximation presented here one finds $\xi^{1.37744}$.

\subsection{Triangular lattice (van de Braak, Caspers and Willemse [20])}

The calculations for the triangular lattice show a great resemblance with those for the linear chain of subsection 2.1. The central problem for this two-dimensional example is the optimal choice for the Kadanoff cells. In fig. 6 a hexagon, with vertices $1,2, \ldots, 6$, is drawn as a representative of the cells used in the calculations of this subsection.

The choice made for the cells, not at all a trivial problem for this example, is determined by the following conditions, partly discussed in the Introduction:

(1) They should contain an odd number of spins $\left(S=\frac{1}{2}\right)$. For a wide range of values of the coupling constants this results in a single Kramers doublet as the lowest state. For special spin systems in which two sublattices may be distinguished, with an antiferromagnetic interaction between nearest neighbours, it was proved by Lieb and Mattis [6] that the lowest level always corresponds with minimal $S$ value. In the more general case only Kramers' theorem can be formulated (cf. appendix B).

(2) The lattice of cells should be isomorphous with the original lattice.

(3) The calculations should be manageable, which condition severely restricts the number of spins per cell.

To make clear why the cells of fig. 6 are chosen the Hamiltonian of the spin lattice is introduced first. Only the case of nearest-neighbour interactions is considered, with restriction to interactions of the isotropic Heisenberg type. Under these conditions the Hamiltonian is

$$
H=J \sum_{\langle i, j\rangle} S_{i} \cdot S_{j}, \quad J>0,
$$

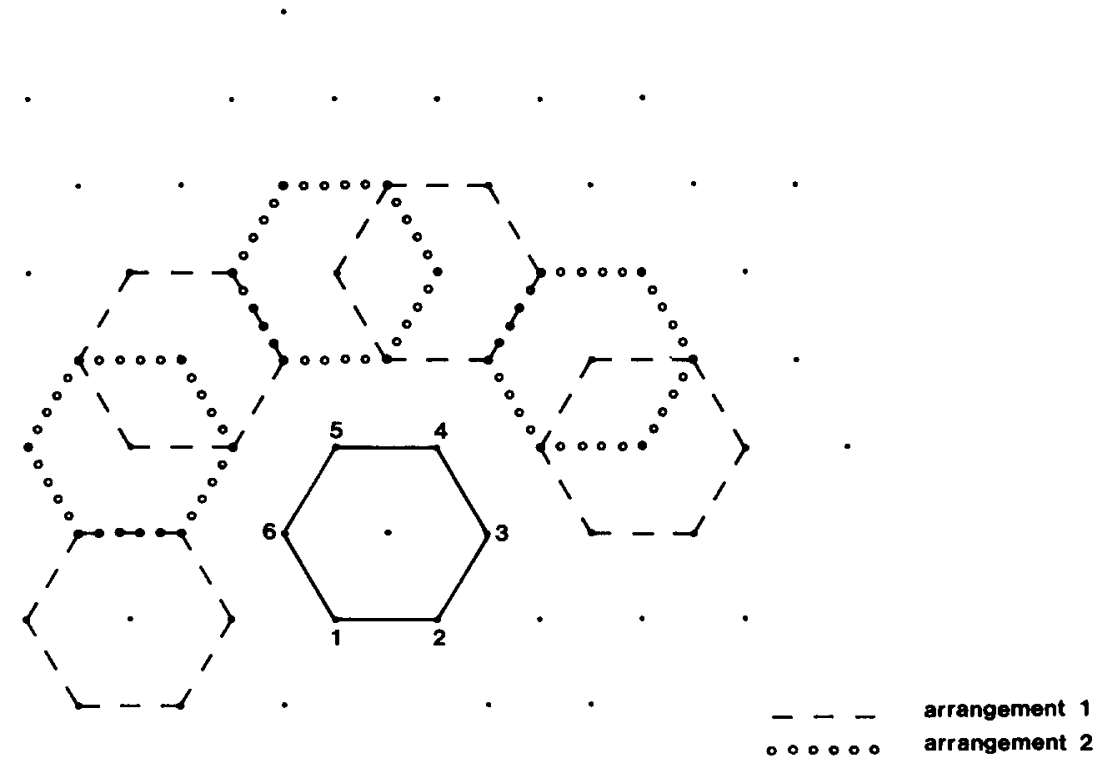

Fig. 6. Kadanoff cells in a triangular lattice [20]. 
the symbol $\langle i, j\rangle$ denoting a pair of nearest neighbours on the triangular lattice. The restriction to antiferromagnetic systems implies $J>0$.

The simplest choice for a Kadanoff cell of triangular symmetry, obeying conditions (2) and (3), is the smallest possible equilateral triangle. But for the given interaction this results in a quartet as the lowest state, i.e. two Kramers doublets (cell spin $S^{(1)}=\frac{1}{2}$ ) of the same energy. The simplest alternative, with one single Kramers doublet as its lowest state, is a hexagon of 7 spins, including the central spin. In fig. 6 this choice is shown as the hexagon with the numbered vertices. Constructing the lattice of cells and starting with an arbitrary site of the original lattice one can place this site in 7 different ways in a cell. There are two different arrangements for the surrounding cells, as is shown in the same figure. Consequently each scaling may be performed in 14 different ways, each way resulting in an isomorphous secular problem for the lattice of cells. This secular problem, apart from the value of its only coupling constant is also isomorphous with the original one (3-1).

For the linear chain and the square and cubic lattices the only freedom is in the choice of the first cell, at least for cells of the simplest type, i.e. rows of consecutive spins, squares and cubes. So far it is not clear if there is any relation between this greater freedom in the renormalization procedure for the triangular lattice and the complications of the geometrical properties of its ground state suggested by Anderson [33] and Fazekas and Anderson [34]. These authors question the existence of sublattices corresponding with a conventional Néel state, in the ground state of the triangular antiferromagnet.

The ground state of a single cell is constructed from a stationary state of the corresponding hexagon without central spin, because the energy of this ring and its total spin are constants of motion for the total cell. An elementary quantum-mechanical calculation, based on the algebraic properties of the spin discussed in appendix A, shows that in the ground state of the cell the spin of the ring $S$ equals 1 , and the total spin $S^{(1)}$ equals $\frac{1}{2}$. The energy is

$$
e_{0}=-\left(2+\frac{1}{2} \sqrt{5}\right) J
$$

The ground state is invariant for the discrete rotations about the hexagonal axis.

Now "scaling" is performed by writing the projected spins of the hexagons in terms of their cell spins. For the spins at positions $1,2, \ldots, 6$ (cf. fig. 6 ) of cell $\boldsymbol{k}$ the projection equals: $\frac{2}{9} \boldsymbol{S}_{\boldsymbol{k}}^{(1)}$. The central spin does not play a role in the interaction between cells in first order. Taking into account that there are three pairs of interacting spins for two neighbouring cells one finds an effective interaction between these cells in their lowest state of the form: $3(2 / 9)^{2} J \boldsymbol{S}_{k}^{(1)} \cdot \boldsymbol{S}_{l}^{(1)}$. The indices $\boldsymbol{k}$ and $l$ for the cells refer to the scaled grid, already discussed in the Introduction.

Making use of the energy per cell $e_{0}$ and the projected interaction between the cells one immediately finds the scaled Hamiltonian $H^{(1)}$

$$
H^{(1)}\left(S_{k}^{(1)}\right)=-\frac{1}{7} N\left(2+\frac{1}{2} \sqrt{5}\right) J I^{(1)}+\frac{4}{27} J \sum_{\langle k, l\rangle} S_{k}^{(1)} \cdot S_{i}^{(1)}
$$

In this example the RG transformation is given by

$$
J^{(1)}=\frac{4}{27} J
$$

Repeated scaling results in the following upper bound for the energy per spin in the lowest state

$$
\epsilon \leq \epsilon^{*}(J)=-\frac{1}{7}\left(2+\frac{1}{2} \sqrt{5}\right)\left[1+\frac{4}{27} \cdot \frac{1}{7}+\left(\frac{4}{27} \cdot \frac{1}{7}\right)^{2}+\cdots\right] J=-\frac{27}{185}\left(2+\frac{1}{2} \sqrt{5}\right) J=-0.45506 J .
$$


This value should be compared with numerical results of the authors already cited [33, 34]. First of all there is the expectation value of the energy per spin for the pure Néel state with three different sublattices with magnetizations at an angle of $120^{\circ}$ to each other. In this Néel state the expectation value of all spins of one sublattice equals $\frac{1}{2}$ in the direction of its magnetization. The expectation value of the energy per spin equals: $-0.375 \mathrm{~J}$ [33]. It may also be looked at as the exact energy of the classical ground state. The same value is found for the so called "pair-bond state" [33] and it is an upper bound for the exact quantum-mechanical value. But the result (3-7) of the renormalization method gives an improvement of more than $20 \%$ as a rigorous upper bound.

Anderson [33] also gives results of rough calculations of the effects of zero-point fluctuations of spin waves. On the basis of techniques used by Stern [35] and Kubo [36] he finds: $(-0.463 \pm 0.007) J$. Calculations for a so called "railroad trestle", as an extrapolation of the results for small systems, give: $(-0.490 \pm 0.005) J[33]$, as a rough estimate for the energy per spin in the triangular lattice. As a further extrapolation from linear chain, via railroad trestle, Anderson gives the value $(-0.54 \pm 0.01) J$. More detailed calculations for very large clusters with fluctuating pair bonds produce the value: $-0.46 J$ [34].

Comparison of the different values in the literature with the result of the renormalization technique given in (3-7), clearly demonstrates the usefulness of this technique, especially for the evaluation of rigorous upper bounds.

\subsection{Linear chain; variational method (van de Braak, Caspers, Gragert and Willemse [22])}

The example of this subsection is again the linear chain with Hamiltonian (1-1). Now a nontrivial unitary operator $u$ will be introduced, whereas the projection operator $P_{0}$ will be the same as in subsection 2.1. The first part of the calculation will be again the determination of the scaled Hamiltonian

$$
P_{0} u^{+} H u P_{0}=H^{(1)}\left(S_{k}^{(1)} ; \gamma\right)
$$

The operator $u$ in this example contains a real parameter $a$. Repeated scaling results in a series of type (7), containing an infinite number of (variable) parameters $a$, one for every scaling phase. Varying this set of parameters a lowest upper bound for the energy per spin will be found.

The projection operator $P_{0}$ corresponds to the lowest Kramers doublets of Kadanoff cells of three spins. Its explicit form may be found in subsection 2.1. The operator $u$ is chosen to be a product of factors pertaining to pairs of neighbouring cells, all factors commuting with each other

$$
\begin{aligned}
& u(a)=\prod_{k} u(k, a), \\
& u(k, a)=\exp \left[\mathrm{i} 2 a\left(S_{3 k+1} \cdot S_{3 k+2}+\frac{1}{4} I\right)\right]=\exp [\mathrm{i} a P(3 k+1,3 k+2)] .
\end{aligned}
$$

In these expressions $a$ is the real parameter already mentioned, and for $a=0$ the trivial case $u(0)=I$ results, which reduces the projected Hamiltonian $H^{(1)}$ to the one of subsection 2.1. The factor $u(k, a)$ couples the $k$ th cell (with spins: $\boldsymbol{S}_{3 k-1}, \boldsymbol{S}_{3 k}$ and $\boldsymbol{S}_{3 k+1}$ ) with the $(k+1)$ rst cell (spins: $\boldsymbol{S}_{3 k+2}, \boldsymbol{S}_{3 k+3}$ and $\boldsymbol{S}_{3 k+4}$ ).

The two-spin operators $P$ are well-known spin-exchange operators $[37,38]$, with the properties

$$
P^{-1}(1,2)=P^{+}(1,2)=P(1,2)
$$




$$
\begin{aligned}
& P(1,2) S_{1} P(1,2)=S_{2}, \quad P(1,2) S_{2} P(1,2)=S_{1}, \\
& {\left[S_{1}, P(1,2)\right]=-\left[S_{2}, P(1,2)\right]=-2 \mathrm{i}\left(S_{1} \times S_{2}\right) .}
\end{aligned}
$$

In the first line the symbol + denotes the hermitean adjoint and consequently the $P$ are hermitean as well as unitary.

From these general properties of spin-exchange operators it follows in a straightforward way

$$
\begin{aligned}
& u(k, a)=\cos a I+2 \mathrm{i} \sin a\left(S_{3 k+1} \cdot S_{3 k+2}+\frac{1}{4} I\right)=\cos a I+\mathrm{i} \sin a P(3 k+1,3 k+2), \\
& {\left[u(a), S^{2}\right]=0, \quad S=\sum_{i} S_{i} .}
\end{aligned}
$$

It should be stressed that $u(a)$ has the full translational symmetry of the lattice of cells. This guarantees the same symmetry for $H^{(1)}$.

The first step in the actual calculation of $H^{(1)}$ is the determination of the transformed Hamiltonian $u^{+} H u$, for which one has to know the transformed operators for the individual spins. The transformed Hamiltonian may then be found on the basis of the general property

$$
u^{+} A B u=u^{+} A u u^{+} B u
$$

$A$ and $B$ being two operators in the spinor space of the total system. The transformed operators for cell $k$ immediately follow from the definition and properties of the operators $u(l, a)$ and $P(3 l+1,3 l+2)$ $(l=k-1, k)$

$$
\begin{aligned}
& u^{+}(a) S_{3 k} u(a)=S_{3 k}, \\
& u^{+}(a) S_{3 k+1} u(a)=\cos ^{2} a S_{3 k+1}+\sin ^{2} a S_{3 k+2}+2 \cos a \sin a\left(S_{3 k+1} \times S_{3 k+2}\right), \\
& u^{+}(a) S_{3 k-1} u(a)=\cos ^{2} a S_{3 k-1}+\sin ^{2} a S_{3 k-2}-2 \cos a \sin a\left(S_{3 k-2} \times S_{3 k-1}\right)
\end{aligned}
$$

Now, after tedious calculations, the transformed operators corresponding to the different terms in $H$, i.e. the internal interactions in one cell $H_{0, k}$ and the interactions between two neighbouring cells $H_{k, k+1}^{\prime}$, both given in (1-2), are found to be

$$
\begin{aligned}
u(a)^{+} H_{0, k} u(a)= & 4\left\{\left[\cos ^{2} a S_{3 k-1}+\sin ^{2} a S_{3 k-2}-2 \cos a \sin a\left(S_{3 k-2} \times S_{3 k-1}\right)\right] \cdot S_{3 k}\right. \\
& +S_{3 k} \cdot\left[\cos ^{2} a S_{3 k+1}+\sin ^{2} a S_{3 k+2}+2 \cos a \sin a\left(S_{3 k+1} \times S_{3 k+2}\right)\right] \\
& +\gamma\left[\cos ^{2} a S_{3 k-1}+\sin ^{2} a S_{3 k-2}-2 \cos a \sin a\left(S_{3 k-2} \times S_{3 k-1}\right)\right] \\
& \left.\cdot\left[\cos ^{2} a S_{3 k+1}+\sin ^{2} a S_{3 k+2}+2 \cos a \sin a\left(S_{3 k+1} \times S_{3 k+2}\right)\right]\right\}, \\
u(a)^{+} H_{k, k+1}^{\prime} u(a)= & 4\left(S_{3 k+1} \cdot S_{3 k+2}+\gamma\left\{S _ { 3 k } \cdot \left[\cos ^{2} a S_{3 k+2}+\sin ^{2} a S_{3 k+1}\right.\right.\right. \\
& \left.-2 \cos a \sin a\left(S_{3 k+1} \times S_{3 k+2}\right)\right]+\left[\cos ^{2} a S_{3 k+1}+\sin ^{2} a S_{3 k+2}\right. \\
& \left.\left.\left.+2 \cos a \sin a\left(S_{3 k+1} \times S_{3 k+2}\right)\right] \cdot S_{3 k+3}\right\}\right) .
\end{aligned}
$$

The final step in the general scheme of section 1 is the projection of the transformed Hamiltonian with a projector $\boldsymbol{P}_{0}$, which, for this example, was introduced in subsection 2.1 . There the projection of the 
linear expressions in terms of spin operators were already given, but here one also has to know the projections of the dyadic forms $\boldsymbol{S}_{3 k} \boldsymbol{S}_{3 k}, \boldsymbol{S}_{3 k} \boldsymbol{S}_{3 k+1}$ etc. .., which appear in the transformed parts of the Hamiltonian: $u(a)^{+} H_{0, k} u(a)$ and $u(a)^{+} H_{k, k+1}^{\prime} u(a)$. For the definition of dyadic forms, the reader is referred to ref. [39]. Elementary quantum-mechanical calculations result in the equivalent dyadic expressions in terms of the cell spins given in table 4 . The general definition of cell spins is given in the Introduction, formula (4).

The symbol 1 in table 4 denotes the unit dyadic with elements: $1_{\alpha \beta}=\delta_{\alpha \beta}$.

Now the projected Hamiltonian $H^{(1)}$ can be calculated and the relevant parameters $\epsilon_{0}, c_{0}$ and $\gamma^{(1)}$ are most conveniently expressed in terms of $x=\cos ^{2} a$,

$$
\begin{aligned}
& H^{(1)}\left(S_{k}^{(1)} ; \gamma\right)=\epsilon_{0}(x, \gamma) N I^{(1)}+c_{0}(x, \gamma) H\left(S_{k}^{(1)} ; \gamma^{(1)}(x, \gamma)\right), \\
& \epsilon_{0}(x, \gamma)=-\frac{4}{3}\left[\gamma+(1-\gamma) x-\frac{1}{4} \gamma x^{2}\right], \\
& c_{0}(x, \gamma)=\frac{4}{9} x[(1+\gamma)-2 \gamma x], \quad x=\cos ^{2} a,
\end{aligned}
$$

the parameter $\gamma^{(1)}(x, \gamma)$ being determined by the $\mathrm{RG}$ transformation

$$
\gamma^{(1)}(x, \gamma)=\frac{1}{3} \gamma \frac{(1-x)(3-x)}{x[(1+\gamma)-2 \gamma x]}
$$

In the scaling procedure there is the freedom to give the parameter $a$ any real value, and consequently $x$ any value between 0 and 1 . The parameter $a$ should be real as a consequence of the restriction to unitary operators $u(a)$.

This scaling procedure may again be repeated an unlimited number of times, resulting in a series of the type given in (7). This time the series depends on an infinite number of parameters $a^{(t)}$ or $x^{(t)}=\cos ^{2} a^{(t)}$, one for every scaling,

$$
\begin{aligned}
& \epsilon(\gamma) \leq \epsilon_{0}(x, \gamma)+\sum_{s=1}^{\infty} \frac{1}{3^{s}}\left[\prod_{i=0}^{s-1} c_{0}\left(x^{(t)}, \gamma^{(t)}\right)\right] \epsilon_{0}\left(x^{(s)}, \gamma^{(s)}\right), \\
& \gamma^{(t)}=\gamma^{(1)}\left(x^{(t-1)}, \gamma^{(t-1)}\right), \quad t=1,2,3, \ldots, \\
& x^{(0)}=x, \quad \gamma^{(0)}=\gamma, \\
& 0 \leq x^{(i)} \leq 1, \quad i=0,1,2,3, \ldots
\end{aligned}
$$

The minimum value of the upper bound in (4-7) will be denoted by $\epsilon^{*}(\gamma)$

$$
\begin{aligned}
& \epsilon(\gamma) \leq \epsilon^{*}(\gamma)=\operatorname{Min}_{x^{(i)}}\left\{\epsilon_{0}(x, \gamma)+\sum_{s=1}^{\infty} \frac{1}{3^{s}}\left[\prod_{t=0}^{s-1} c_{0}\left(x^{(t)}, \gamma^{(t)}\right)\right] \epsilon_{0}\left(x^{(s)}, \gamma^{(s)}\right)\right\}, \quad x^{(0)}=x . \\
& \text { Table } 4 \\
& \text { Projected operators for cell } k[18,22]
\end{aligned}
$$

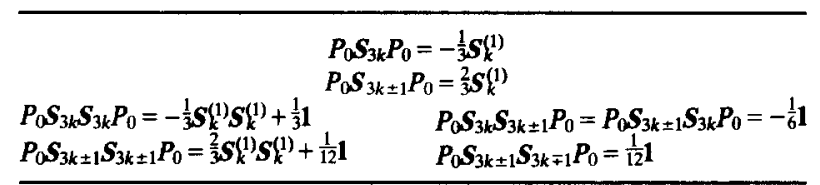


Making the hypothesis that the minimum is attained for values of the $x^{(i)}$ for which the coefficients $c_{0}\left(x^{(t)}, \gamma^{(t)}\right) \geq 0$, one easily derives the following equation for $\epsilon^{*}(\gamma)$

$$
\epsilon^{*}(\gamma)=\operatorname{Min}_{x, c_{0}(x, \gamma) \geq 0}\left[\epsilon_{0}(x, \gamma)+\frac{1}{3} c_{0}(x, \gamma) \epsilon^{*}\left(\gamma^{(1)}(x, \gamma)\right)\right]
$$

So far no attention has been paid to the possible convergence of the series for $\epsilon^{*}(\gamma)$. The exact solution of (4-7.1), however, corresponds to a geometrical series for every $\gamma$, apart from an additive constant. The convergence of this series then follows in an elementary way.

One may also raise the question whether or not the RG equation for the minimum, $\gamma^{(t)}=$ $\gamma^{(1)}\left(x^{(t-1)}, \gamma^{(t-1)}\right)$, gives a Kramers doublet as the lowest level for a cell, if $\gamma^{(t-1)}$ corresponds to such an arrangement of the levels, i.e. $\gamma^{(t)}<1$ for $\gamma^{(t-1)}<1$. However, even for one or more $\gamma^{(t)}>1$, (4-7.1) still gives an upper bound and the scaling method is not absolutely restricted by the order of the levels, but one may doubt about its usefulness if the lowest level is not a Kramers doublet.

The formula (4-7.2) certainly gives an upper bound for $\epsilon(\gamma)$, because it corresponds to a special choice for the $x^{(i)}$ in (4-7.1). In the mathematical determination of $\epsilon^{*}(\gamma)$ the condition $c_{0}(x, \gamma) \geq 0$ was omitted, in first instance. It turned out, however, that the solution found in this way obeyed this constraint.

The solution of (4-7.2) was found in two steps: First it was derived in the neighbourhood of $\gamma=0$, which gives a linear expression in $\gamma$. This linear expression was then used as a first step in an iteration procedure. This procedure, performed on a DEC10 electronic computer, turned out to be rapidly converging and its result could be represented in an analytic way. This analytic solution was found to obey the equation for $\epsilon^{*}(\gamma)$ exactly, the minimum being conceived as a relative one.

From the expression for $\gamma^{(1)}(x, \gamma)$ in (4-6) one immediately derives that $\gamma^{(1)}(x, 0)=0(x \neq 0)$ and excluding the value $x=0$ one finds the following equation for $\epsilon^{*}(0)$

$$
\epsilon^{*}(0)=\operatorname{Min}_{x \neq 0}\left[\epsilon_{0}(x, 0)+\frac{1}{3} c_{0}(x, 0) \epsilon^{*}(0)\right]
$$

which can be solved in an elementary way to give

$$
\epsilon^{*}(0)=-\frac{36}{23}, \quad \hat{x}(0)=1 .
$$

Here the function $\hat{x}(y)$ is introduced, representing the value of $x$ for which the minimum in (4-7.2) is reached. For $\gamma=0$ there is a boundary extremum for the variable $x=1$ and making the hypothesis $\hat{x}(\gamma) \equiv 1$ in a finite interval with $\gamma=0$ as an interior point one finds the solution

$$
\epsilon^{*}(\gamma)=\epsilon_{0}(1, \gamma)+\frac{1}{3} c_{0}(1, \gamma) \epsilon^{*}(0)=-\frac{36}{23}+\frac{13}{23} \gamma
$$

This expression may be used as a zero-order solution for all (real) values of $\gamma$ and the equation (4-7.2) may be solved numerically by means of iteration. This procedure gave two regions in which there exists a boundary extremum:

$$
\begin{array}{ll}
\gamma \leqq 0.59: & \hat{x}(\gamma) \equiv 1 \text { and } \\
\gamma \geq 0.81: & \hat{x}(\gamma) \equiv 0 .
\end{array}
$$


Table 5

Analytic solution for $\epsilon^{*}(\gamma)[22]$

\begin{tabular}{lll}
\hline$-\infty<\gamma<\gamma_{1}=\frac{1458}{2459}$ & $\epsilon^{*}(\gamma)=-\frac{36}{23}+\frac{13}{23} \gamma$, & $\hat{x}(\gamma)=1$ \\
$\gamma_{1}<\gamma<\gamma_{2}=0.77487$ & $\epsilon^{*}(\gamma)=-\frac{26244}{35351} \frac{1}{\gamma}+\frac{33192}{35351}-\frac{4428220}{2863431} \gamma$, & $\hat{x}(\gamma)=\frac{1458-922 \gamma}{1537 \gamma}$ \\
$\gamma_{2}<\gamma<\gamma_{3}=\frac{69}{85}$ & $\epsilon^{*}(\gamma)=-\frac{92}{53} \frac{1}{\gamma}+\frac{680}{159}-\frac{46072}{10971} \gamma$, & $\hat{x}(\gamma)=\frac{138-170 \gamma}{53 \gamma}$ \\
$\gamma_{3}<\gamma<\infty$ & $\epsilon^{*}(\gamma)=-\frac{36}{23} \gamma$, & $\hat{x}(\gamma)=0$ \\
\hline
\end{tabular}

On the basis of this knowledge the analytic solution could be reconstructed in a straightforward way, as will be discussed shortly. The complete solution is given in table 5. The continuous curve for $\epsilon^{*}(\gamma)$ consists of two straight lines and two hyperbolic parts. The values $\gamma_{1}, \gamma_{2}$ and $\gamma_{3}$ correspond with boundaries between two adjacent parts, $\gamma_{2}$ being the largest root of the quadratic equation:

$$
1899143 \gamma^{2}-2389392 \gamma+711180=0 \text {. }
$$

The function $\hat{x}(\gamma)$ is continuous with the exception of the point $\gamma=\gamma_{2}$, for which there is a finite discontinuity. A rather lengthy, but elementary argument shows that the same is true for $\mathrm{d} \epsilon^{*} / \mathrm{d} \gamma$. The curve $\epsilon^{*}(\gamma)$ is shown in fig. 1, together with the results for the linear chain found with other approximations. Having found the analytic solution of table 5 it is easy to prove that this solution obeys eq. (4-7.2), in such a sense that for each of the four parts of the curve, $\hat{x}(\gamma)$ represents a local minimum, which is a boundary extremum for $\gamma<\gamma_{1}$ and $\gamma>\gamma_{3}$. The function $\gamma^{(1)}(\hat{x}(\gamma), \gamma)$ maps the interval $\left[\gamma_{1}, \gamma_{2}\right]$ on an interval $\left[0, \bar{\gamma}_{2}\right]$, which is part of $\left[0, \gamma_{1}\right]$, whereas it maps $\left[\gamma_{2}, \gamma_{3}\right]$ on $\left[\overline{\bar{\gamma}}_{2}, \infty\right]$, the latter being part of $\left[\gamma_{3}, \infty\right]$. Naively starting from the analytic solution in the interval $\left[0, \gamma_{1}\right]$ one could construct a series of adjacent intervals for $\gamma \geq 0$, each mapped on its left neighbour by the algebraic solution of the minimum problem (4-7.2)

$$
\frac{\partial}{\partial x}\left[\epsilon_{0}(x, \gamma)+\frac{1}{3} c_{0}(x, \gamma) \epsilon^{*}\left(\gamma^{(1)}(x, \gamma)\right)\right]=0, \quad 0 \leq x \leq 1
$$

which defines the function $\hat{x}(\gamma)$. The endpoints $\gamma_{1}, \gamma_{(2)}, \gamma_{(3)}, \ldots$ of the intervals are mapped on each other, i.e. 0 is the image of $\gamma_{1}, \gamma_{1}$ of $\gamma_{(2)}$, etc. etc.

This was indeed the first approach to the solution of (4-7.2) but it turned out, in the numerical calculations, that for $\gamma>\gamma_{2}$ this analytic solution does not represent the absolute minimum. One can construct an analogous procedure starting from the interval $\left[\gamma_{3}, \infty\right]$ and this results in a mapping to the right. The last procedure, however, gives a lower value for $\epsilon^{*}(\gamma)$, for $\gamma>y_{2}$.

In table 6 numerical values for $\epsilon^{*}$ are given, based on the formulas of table 5. A comparison with the results of subsections 2.1 and 2.5 is given in section 4 .

\subsection{Linear chain; second order (van de Braak, Caspers, de Lange and Willemse [18, 21])}

All the information about the secular problem for the linear chain has been given already in subsection 2.1. Also in this subsection nearest neighbour and next-nearest neighbour interactions are taken into consideration. Once again the analysis is based on Kadanoff cells of three spins and the total 
Table 6

\begin{tabular}{|c|c|c|c|c|c|}
\hline \multicolumn{6}{|c|}{$\epsilon^{*}$ as a function of $\gamma$ [22] } \\
\hline$\gamma$ & $\epsilon^{*}$ & $\gamma$ & $\epsilon^{*}$ & $\gamma$ & $\epsilon^{*}$ \\
\hline-1.00 & -2.13043 & 0.25 & -1.42391 & $\gamma_{2}=0.77487$ & -1.21746 \\
\hline-0.95 & -2.10217 & 0.30 & -1.39565 & 0.78 & -1.22428 \\
\hline-0.90 & -2.07391 & 0.35 & -1.36739 & 0.79 & -1.23810 \\
\hline-0.85 & -2.04565 & 0.40 & -1.33913 & 0.80 & -1.25263 \\
\hline-0.80 & -2.01739 & 0.45 & -1.31087 & 0.81 & -1.26783 \\
\hline-0.75 & -1.98913 & 0.50 & -1.28261 & $\gamma_{3}=\frac{69}{85}$ & -1.27059 \\
\hline-0.70 & -1.96087 & 0.55 & -1.25435 & 0.82 & -1.28348 \\
\hline-0.65 & -1.93261 & $\gamma_{1}=\frac{1458}{2459}$ & -1.23009 & 0.83 & -1.29913 \\
\hline-0.60 & -1.90435 & 0.60 & -1.22626 & 0.84 & -1.31478 \\
\hline-0.55 & -1.87609 & 0.61 & -1.22144 & 0.85 & -1.33043 \\
\hline-0.50 & -1.84783 & 0.62 & -1.21728 & 0.86 & -1.34609 \\
\hline-0.45 & -1.81957 & 0.63 & -1.21374 & 0.87 & -1.36174 \\
\hline-0.40 & -1.79130 & 0.64 & -1.21079 & 0.88 & -1.37739 \\
\hline-0.35 & -1.76304 & 0.65 & -1.20841 & 0.89 & -1.39304 \\
\hline-0.30 & -1.73478 & 0.66 & -1.20657 & 0.90 & -1.40870 \\
\hline-0.25 & -1.70652 & 0.67 & -1.20525 & 0.91 & -1.42435 \\
\hline-0.20 & -1.67826 & 0.68 & -1.20442 & 0.92 & -1.44000 \\
\hline-0.15 & -1.65000 & 0.69 & -1.20406 & 0.93 & -1.45565 \\
\hline-0.10 & -1.62174 & 0.70 & -1.20415 & 0.94 & -1.47130 \\
\hline-0.05 & -1.59348 & 0.71 & -1.20468 & 0.95 & -1.48696 \\
\hline 0 & -1.56522 & 0.72 & -1.20562 & 0.96 & -1.50261 \\
\hline 0.05 & -1.53696 & 0.73 & -1.20696 & 0.97 & -1.51826 \\
\hline 0.10 & -1.50870 & 0.74 & -1.20868 & 0.98 & -1.53391 \\
\hline 0.15 & -1.48043 & 0.75 & -1.21077 & 0.99 & -1.54957 \\
\hline 0.20 & -1.45217 & 0.76 & -1.21321 & 1.00 & -1.56522 \\
\hline & & 0.77 & -1.21599 & & \\
\hline
\end{tabular}

Hamiltonian (1-1) is subdivided according to (1-2). Consequently the solution of the secular problem in zero-order is given by (1-3).

The important difference with subsection 2.1 lays in the choice of the projection operator $P_{1}$ and the corresponding unitary operator $u$, which are given now by Rayleigh-Schrödinger perturbation theory in second order. The perturbation problem is degenerate, but it can be solved formally along the same lines as a non-degenerate problem. The formulation of the non-degenerate problem and its solution for the energy is given in many textbooks: Here the reader is referred to Thouless [40] chapter IV, formulas (4.7) and (4.8). The solution (5-5) is discussed in elementary texts under the heading: Removal of degeneracy in second order [41].

For the degenerate problem the unperturbed ground state may be represented by the set $|0, p\rangle$ and the exact ground state is one of a set $|p\rangle$. Both sets are composed of $2^{N / 3}$ states, being the total number of Kramers states for cells of three spins. The states $|p\rangle$ and their corresponding energy eigenvalues $E_{0}$ are solutions of the secular problem given by

$$
\begin{aligned}
& |p\rangle=|0, p\rangle+\frac{I-P_{0}}{W_{0}-H_{0}}\left(H^{\prime}-E_{0}+W_{0}\right)|p\rangle \\
& \left(E_{0}-W_{0}\right) \delta_{p q}=\left\langle 0, p\left|H^{\prime}\right| q\right\rangle .
\end{aligned}
$$


These formulas are a straightforward generalization of (4.7) and (4.8) of ref. [40]. Here $I$ is the identity operator and $W_{0}$ the unperturbed ground-state energy given by $(1-3)$, i.e.: $W_{0}=(N / 3) e_{0}(\gamma)$. The operator $P_{0}$ projects on the set of states $|0, p\rangle$ and $H_{0}$ and $H^{\prime}$ are the parts of the Hamiltonian defined in (1-2). The states $|0, p\rangle$ are linear combinations of the states $\left|0, m_{k}\right\rangle$ chosen in such a way that $\left\langle 0, p\left|H^{\prime}\right| q\right\rangle$ is diagonal. This last condition defines a secular problem that gives the eigenvalues $E_{0}$, the lowest of which being the (exact) ground-state energy. The first formula, defining $|p\rangle$ in terms of $|0, p\rangle$ gives, in principle, the unitary operator $u$, but it is not necessary for the scaling procedure to give an explicit expression for this operator.

In the approximation in second order, used in this subsection, the state $|q\rangle$ in the second formula (5-1.1), is replaced by its first-order approximation, which follows from the first formula. This results in the secular problem

$$
\left(E_{0}-W_{0}\right) \delta_{p q}=\left\langle 0, p\left|H^{\prime}\right| 0, q\right\rangle+\left\langle 0, p\left|H^{\prime} \frac{I-P_{0}}{W_{0}-H_{0}} H^{\prime}\right| 0, q\right\rangle .
$$

The states $|0, p\rangle,|0, q\rangle, \ldots$ being linear combinations of the states $\left|0, m_{k}\right\rangle$, this secular problem may be conveniently formulated in terms of the cell-spin operators $\boldsymbol{S}_{k}^{(1)}$. These cell-spin operators were defined generally in the Introduction, formula (4). Here the cell index $\boldsymbol{k}$ has to be replaced by $k$.

From formula (5-1.2) it immediately follows that the eigenvalues $E_{0}$, in this approximation, are the eigenvalues of a Hamiltonian $H^{(1)}$ defined by

$$
H^{(1)}\left(S_{k}^{(1)} ; \gamma\right)=W_{0} I^{(1)}+P_{0} H^{\prime} P_{0}+P_{0} H^{\prime} \frac{I-P_{0}}{W_{0}-H_{0}} H^{\prime} P_{0}
$$

The evaluation of $H^{(1)}$ results again in an expression of the type (5), i.e. the Hamiltonian is properly scaled: The original Hamiltonian is reproduced for the cells, apart from the value of the parameter $\gamma$. This "scaling" is a consequence of a suitable combination of the original Hamiltonian and the approximation method. If the perturbation calculus is performed up to and including the third order, this would spoil this "scaling" property, because other interaction terms than the scalar spin coupling between nearest and next-nearest neighbour cells would appear in $H^{(1)}$.

Because $H^{(1)}$ is determined by second-order perturbation calculus it generally does not give an upper bound for the energy of the ground state. Repeated scaling in this example results in a series of the type given in formula (8). The method for the evaluation of $H^{(1)}$ is the parallel of the spin-Hamiltonian formalism for electron-spin resonance, extensively discussed in the literature (Pryce [9], Abragam and Pryce [10], Bleany and Stevens [11] and Caspers [12]).

In the actual evaluation of $H^{(1)}$ for the linear chain only the third term: $P_{0} H^{\prime}\left\{\left(I-P_{0}\right) /\left(W_{0}-H_{0}\right)\right\} H^{\prime} P_{0}$ has to be determined here, because the sum of the first two terms has been given already in (1-5), i.e. the first-order approximation of $H^{(1)}$.

The different parts of this third term, representing contributions of second order in $H^{\prime}$, correspond with different excited states of individual cells. These excitations are effected by the terms $H_{k, k+1}^{\prime}$ of $(1-2)$, coupling neighbouring cells.

So the intermediate states, selected by the projection operator $I-P_{0}$, can only correspond with one excited cell or with a pair of neighbouring cells, both in excited states.

For the calculations it is necessary to give all the stationary states of the individual cells for the Hamiltonian of zero order $H_{0}$, also given in (1-2). The lowest Kramers doublet was already given in subsection 2.1, but here a complete list of stationary states is shown in table 7, omitting the cell index $k$. For this set $m$, representing $m_{k}$ for the $k$ th cell, is chosen to be a good quantum number. 
Table 7

The stationary states of a cell [18]

\begin{tabular}{|c|c|c|c|}
\hline & $m$ & & $e(\gamma)$ \\
\hline$\left|\frac{3}{2}, m\right\rangle$ & $\begin{array}{r}\frac{3}{2} \\
\frac{1}{2} \\
-\frac{1}{2} \\
-\frac{3}{2}\end{array}$ & $\begin{array}{l}|+++\rangle \\
\frac{1}{\sqrt{3}}[|+-+\rangle+(|-++\rangle+|++-\rangle)] \\
\frac{1}{\sqrt{3}}[|-+-\rangle+(|+--\rangle+|--+\rangle)] \\
|---\rangle\end{array}$ & $2+\gamma$ \\
\hline$\left|\frac{1}{2}-, m\right\rangle$ & $\begin{array}{r}\frac{1}{2} \\
-\frac{1}{2}\end{array}$ & $\left.\begin{array}{c}\frac{1}{\sqrt{2}}(|-++\rangle-|++-\rangle) \\
-\frac{1}{\sqrt{2}}(|+--\rangle-|--+\rangle)\end{array}\right\}$ & $-3 \gamma$ \\
\hline$\left|\frac{1}{2}+, m\right\rangle$ & $\begin{array}{r}\frac{1}{2} \\
-\frac{1}{2}\end{array}$ & $\begin{array}{l}-\frac{1}{\sqrt{6}}[2|+-+\rangle-(|-++\rangle+|++-\rangle)] \\
\frac{1}{\sqrt{6}}[2|-+-\rangle-(|+--\rangle+|--+\rangle)]\end{array}$ & $-4+\gamma=e_{0}(\gamma)$ \\
\hline
\end{tabular}

The nomenclature used for the kets $|+--\rangle$ etc., was already introduced in subsection 2.1 . The states fall apart into three groups corresponding with two doublets: $\left|\frac{1}{2}+, m\right\rangle$ and $\left|\frac{1}{2}-, m\right\rangle$ and one quartet: $\left|\frac{3}{2}, m\right\rangle$. For the two doublets the total spin $S^{(1)}$ for a cell equals $\frac{1}{2}$ and for the quartet $S^{(1)}=\frac{3}{2}$. The energy is given in the last column and it is clear that the lowest level corresponds with $\left.\mid \frac{1}{2}+, m\right)$ for $\gamma<1$.

Calculations are restricted to $\gamma<1$, corresponding with a well-deffned lowest doublet, also after repeated scaling, i.e. $\gamma^{(t)}<1, t=1,2, \ldots$ (cf. formulas (6) and (8)). The \pm sign in the kets $\left|\frac{1}{2} \pm, m\right\rangle$ indicates the symmetry character of the states for the interchanging of the spins at positions $3 k-1$ and $3 k+1$ in cell $k$.

The determination of the second-order part of $H^{(1)}$ goes in two steps. First an expression for $\left\{\left(I-P_{0}\right) /\left(W_{0}-H_{0}\right)\right\} H^{\prime} P_{0}$ is given in terms of one- and two-cell excitations

$$
\begin{aligned}
\frac{I-P_{0}}{W_{0}-H_{0}} H^{\prime} P_{0}= & \sum_{k}\left\{\frac{1}{-4+4 \gamma} \bar{P}_{1 / 2-}(k)+\frac{1}{-6} \bar{P}_{3 / 2}(k)+\frac{1}{2(-4+4 \gamma)} \bar{P}_{1 / 2-, 1 / 2-}(k, k+1)\right. \\
& +\frac{1}{-10+4 \gamma}\left[\bar{P}_{1 / 2-, 3 / 2}(k, k+1)+\bar{P}_{3 / 2,1 / 2-}(k, k+1)\right] \\
& \left.+\frac{1}{2(-6)} \bar{P}_{3 / 2,3 / 2}(k, k+1)\right\} H^{\prime} \prod_{k^{\prime}} P_{1 / 2+}\left(k^{\prime}\right)
\end{aligned}
$$

In formula (5-5.1) the projection operator $P_{1 / 2+}\left(k^{\prime}\right)$ projects on the lowest doublet for cell $k^{\prime}$. The projection operators $\bar{P}_{1 / 2}(k)$ and $\bar{P}_{3 / 2}(k)$ project on the product space of the lowest doublets for all cells, with the exception of cell $k$, for which it projects on the doublet $\left|\frac{1}{2}-, m_{k}\right\rangle$, respectively the quartet $\left|\frac{3}{2}, m_{k}\right\rangle$. The $\bar{P}_{1 / 2-1 / 2-}(k, k+1), \bar{P}_{1 / 2-, 3 / 2}(k, k+1)$, etc. ... correspond in an analogous way to double excitations of the neighbour cells $k$ and $k+1$. The energy denominators in (5-5.1) immediately follow from table 7 . 
For further evaluation of (5-5.1) formulas are given in table 8 for the projected operators:

$$
P_{1 / 2+}(k) S_{3 k} P_{1 / 2+}(k), \quad P_{1 / 2+}(k) S_{3 k \pm 1} P_{1 / 2+}(k), \quad P_{1 / 2-}(k) S_{3 k} P_{1 / 2+}(k), \ldots
$$

the projection operators $P_{1 / 2}(k)$ and $P_{3 / 2}(k)$ being defined in an analogous way as the $P_{1 / 2+}(k)$.

It should be stressed that the effective operators in the right-hand members in table 8 are chosen in such a way that they select the proper final multiplet, $\left\{\frac{1}{2}+, m_{k}\right\rangle,\left|\frac{1}{2}-, m_{k}\right\rangle$ or $\left|\frac{3}{2}, m_{k}\right\rangle$. The $\boldsymbol{S}_{k}^{(1)}$ again corresponds with the cell spin of cell $k$.

With the help of table 8 and the expression for $H^{\prime}$ in (1-2) the different projected parts of $H^{\prime}$ are easily evaluated. The results are listed in table 9.

In the second step of the determination of $H^{(1)}$ in second order one has to determine projected operators like

$$
\prod_{k^{\prime \prime}} P_{1 / 2+}\left(k^{\prime \prime}\right) H^{\prime} \bar{P}_{1 / 2-}(k) \frac{1}{W_{0}-H_{0}} \bar{P}_{1 / 2-}(k) H^{\prime} \prod_{k^{\prime}} P_{1 / 2+}\left(k^{\prime}\right), \text { etc. } \ldots,
$$

representing different intermediate excitations. With the results of table 9 the different expressions (5-5.2) may be written in terms of the spin operators and the projection operator for the unperturbed ground state: $\Pi_{k} P_{1 / 2+}(k)$.

Because of this projection the expressions are functions of the cell spins $\boldsymbol{S}_{k}^{(1)}$ alone, apart from a possible constant.

For reasons of time-reversal symmetry and spherical symmetry of the original Hamiltonian (1-2), only bilinear terms of the form $c \boldsymbol{S}_{k}^{(1)} \cdot \boldsymbol{S}_{k+1}^{(1)}$ and $d \boldsymbol{S}_{k}^{(1)} \cdot \boldsymbol{S}_{k+2}^{(1)}$ appear in the final expression for $H^{(1)}$, hermitean forms quadratic in the components of one single spin $\boldsymbol{S}_{k}^{(1)}$ giving a constant.

In the reduction of the complicated expressions (5-5.2) one also needs the equivalent dyadic forms of table 4 , and the algebraic relation

$$
\left(\boldsymbol{S}_{k}^{(1)} \cdot \boldsymbol{S}_{k+1}^{(1)}\right)^{2}=\frac{3}{16}-\frac{1}{2}\left(\boldsymbol{S}_{k}^{(1)} \cdot \boldsymbol{S}_{k+1)}^{(1)}\right)
$$

$\bar{P}_{1 / 2-}(k) H^{\prime} \Pi_{k^{\prime}} P_{1 / 2+}\left(k^{\prime}\right)=\frac{2}{3}(2-\gamma)\left(S_{3 k-1}-S_{3 k+1}\right) \cdot\left[\left(S_{3 k-4}+S_{3 k-3}+S_{3 k-2}\right)-\left(S_{3 k+2}+S_{3 k+3}+S_{3 k+4}\right)\right] \Pi_{k^{\prime}} \cdot P_{1 / 2+}\left(k^{\prime}\right)$,

$\bar{P}_{3 / 2}(k) H^{\prime} \Pi_{k^{\prime}} P_{1 / 2+}\left(k^{\prime}\right)=\frac{2}{9}(-2+5 y)\left(S_{3 k-1}+4 S_{3 k}+S_{3 k+1}\right) \cdot\left[\left(S_{3 k-4}+S_{3 k-3}+S_{3 k-2}\right)+\left(S_{3 k+2}+S_{3 k+3}+S_{3 k+4}\right)\right] \Pi_{k^{\prime}} P_{1 / 2+}\left(k^{\prime}\right)$,

$\bar{P}_{1 / 2-1 / 2}(k, k+1) H^{\prime} \Pi_{k^{\prime}} P_{1 / 2+}\left(k^{\prime}\right)=-\left(S_{3 k-1}-S_{3 k+1}\right) \cdot\left(S_{3 k+2}-S_{3 k+4}\right) \Pi_{k^{\prime}} P_{1 / 2+}\left(k^{\prime}\right)$,

$\bar{P}_{1 / 2-3 / 2}(k, k+1) H^{\prime} \Pi_{k^{\prime}} P_{1 / 2+}\left(k^{\prime}\right)=\frac{1}{3}(1-2 \gamma)\left(S_{3 k-1}-S_{3 k+1}\right) \cdot\left(S_{3 k+2}+4 S_{3 k+3}+S_{3 k+4}\right) \Pi_{k^{\prime}} P_{1 / 2+}\left(k^{\prime}\right)$,

$\bar{P}_{3 / 2,1 / 2}(k, k+1) H^{\prime} \Pi_{k^{\prime}} P_{1 / 2+}\left(k^{\prime}\right)=-\frac{1}{3}(1-2 \gamma)\left(S_{3 k-1}+4 S_{3 k}+S_{3 k+1}\right) \cdot\left(S_{3 k+2}-S_{3 k+4}\right) \Pi_{k^{\prime}} P_{1 / 2}+\left(k^{\prime}\right)$,

$\bar{P}_{3 / 2,3 / 2}(k, k+1) H^{\prime} \Pi_{k^{\prime}} P_{1 / 2+}\left(k^{\prime}\right)=\frac{1}{q}(1-4 \gamma)\left(S_{3 k-1}+4 S_{3 k}+S_{3 k+1}\right) \cdot\left(S_{3 k+2}+4 S_{3 k+3}+S_{3 k+4}\right) \Pi_{k^{\prime}} P_{1 / 2+}\left(k^{\prime}\right)$. 
Making use of all the foregoing results the second-order part of $H^{(1)}$ may be written

$$
\begin{aligned}
H_{2}^{(1)}= & {\left[-\frac{3-16 \gamma+22 \gamma^{2}}{\left.27-\frac{19-16 \gamma+4 \gamma^{2}}{72(1-\gamma)}-\frac{2}{9} \frac{1-4 \gamma+4 \gamma^{2}}{5-2 \gamma}\right] \frac{N}{3} I^{(1)}}\right.} \\
& +\left[-\frac{2}{81}\left(5-24 \gamma+28 \gamma^{2}\right)+\frac{19-16 \gamma+4 \gamma^{2}}{27(1-\gamma)}-\frac{8}{27} \frac{1-4 \gamma+4 \gamma^{2}}{5-2 \gamma}\right] \sum_{k} S_{k}^{(1)} \cdot S_{k+1}^{(1)} \\
& +\left[-\frac{8}{243}\left(4-20 \gamma+25 \gamma^{2}\right)+\frac{2}{27} \frac{4-4 \gamma+\gamma^{2}}{1-\gamma}\right] \sum_{k} S_{k}^{(1)} \cdot S_{k+2}^{(1)} .
\end{aligned}
$$

In order to find the total expression for the scaled Hamiltonian up to and including second-order terms, one has to combine $H_{2}^{(1)}$ with the parts of zeroth and first order given in (1-5), which results in

$$
\begin{aligned}
H^{(1)}\left(S_{k}^{(1)} ; \gamma\right)= & {\left[-\frac{111-43 \gamma+22 \gamma^{2}}{27}-\frac{19-16 \gamma+4 \gamma^{2}}{72(1-\gamma)}-\frac{2}{9} \frac{1-4 \gamma+4 \gamma^{2}}{5-2 \gamma}\right] \frac{N}{3} I^{(1)} } \\
& +\left[\frac{2}{81}\left(67-48 \gamma-28 \gamma^{2}\right)+\frac{19-16 \gamma+4 \gamma^{2}}{27(1-\gamma)}-\frac{8}{27} \frac{1-4 \gamma+4 \gamma^{2}}{5-2 \gamma}\right] \sum_{k} S_{k}^{(1)} \cdot S_{k+1}^{(1)} \\
& +\left[-\frac{8}{243}\left(4-20 \gamma+25 \gamma^{2}\right)+\frac{2}{27} \frac{4-4 \gamma+\gamma^{2}}{1-\gamma}\right] \sum_{k} S_{k}^{(1)} \cdot S_{k+2}^{(1)} .
\end{aligned}
$$

This expression is of the proper renormalized form (5), because it may be completely expressed in terms of the original Hamiltonian (1-1) for the cell spins $\boldsymbol{S}_{k}^{(1)}$. Typical for the form (5) are an additive and multiplicative constant, respectively denoted by $\epsilon_{0} N I^{(1)}$ and $c_{0}$, and a shifted value for the parameter that determines the ratio between next-nearest and nearest-neighbour interactions. The last parameter is denoted by $\gamma^{(1)}$ for the scaled system.

The values of $\epsilon_{0}, c_{0}$ and $\gamma^{(1)}$ depend on the parameter $\gamma$ of the original Hamiltonian (1-1) and they follow from their definition in formula (5) together with the expression for $H^{(1)}$ in (5-5.3).

Expressions for $\epsilon_{0}(\gamma)$ and $c_{0}(\gamma)$ are

$$
\begin{aligned}
& \epsilon_{0}(\gamma)=\frac{1}{3}\left[-\frac{111-43 \gamma+22 \gamma^{2}}{27}-\frac{19-16 \gamma+4 \gamma^{2}}{72(1-\gamma)}-\frac{2}{9} \frac{1-4 \gamma+4 \gamma^{2}}{5-2 \gamma}\right] \\
& c_{0}(\gamma)=\frac{1}{4}\left[\frac{2}{81}\left(67-48 \gamma-28 \gamma^{2}\right)+\frac{19-16 \gamma+4 \gamma^{2}}{-27(1-\gamma)}-\frac{8}{27} \frac{1-4 \gamma+4 \gamma^{2}}{5-2 \gamma}\right]
\end{aligned}
$$

whereas the so-called renomalization-group (RG) transformation is defined by

$$
\gamma^{(1)}(\gamma)=\frac{1}{4 c_{0}(\gamma)}\left[-\frac{8}{243}\left(4-20 \gamma+25 \gamma^{2}\right)+\frac{2}{27} \frac{4-4 \gamma+\gamma^{2}}{1+\gamma}\right]=\Gamma(\gamma)
$$

These three equations fully determine the series (8) for the approximation of $\epsilon(\gamma)$, which, in this example, is the energy per spin in the ground state of a linear chain of spins $\frac{1}{2}$.

The equation (5-6) for the RG transformation defines a stable point as a solution of the equation

$$
\gamma=\Gamma(\gamma)
$$


or,

$$
336 \gamma^{5}-1216 \gamma^{4}-188 \gamma^{3}+3006 \gamma^{2}-2273 \gamma+200=0 .
$$

Numerical calculations have been restricted to the interval $|\gamma|<1$, for which the lowest state for an isolated cell is the doublet $\left|\frac{1}{2}+, m\right\rangle$. For these values of $\gamma$ the RG equation (5-6) gives a $\gamma^{(1)}$ in the same interval. Consequently all the parameters $\gamma^{(s)}$ in the series (8) obey the inequality $\left|\gamma^{(s)}\right|<1$.

There is only one solution of (5-6.1) in this interval, representing the stable point

$$
\gamma^{*}=0.10146
$$

for the series (8). Graphically it may be found as an intersection of the curve for $\Gamma(\gamma)$ and a straight line, both drawn in fig. 7. The function $\Gamma(\gamma)$ has a slope between 0 and 1 , for $|\gamma| \leq 1$; consequently repeated scaling gives a row of parameter values $\gamma^{(1)}, \gamma^{(2)}, \gamma^{(3)}, \ldots$, converging to $\gamma^{*}$. Now it is possible to determine $\epsilon(\gamma)$ from the general formula (8) with $n=3$, and the explicit expressions for $\epsilon_{0}(\gamma), c_{0}(\gamma)$ and $\gamma^{(1)}(\gamma)$, together with the parameter values $\gamma^{(2)}, \gamma^{(3)}, \ldots$. Convergence of the series is guaranteed by the fact that its terms, for every $\gamma$ value, converge to those of a geometrical series, for $s \rightarrow \infty$. Numerical results were found with a Wang 500 desk calculator. Table 10 gives the results for $\epsilon(\gamma)$ in the interval $-1 \leq \gamma<1$.

The curve for $\epsilon(\gamma)$ found along these lines is also drawn in fig. 1, together with the approximations of subsections 2.1 and 2.4. For $\gamma=1, \epsilon(\gamma)$ diverges in the approximation of this subsection because the distance between the two doublets of one cell goes to zero. It makes sense to devote a special section to the discussion of the merits of the renormalization technique for the determination of the ground-state energy of antiferromagnetic lattices, especially in the case of the linear chain, for which three different applications of this technique have been used. This discussion is given in the final section 4 .

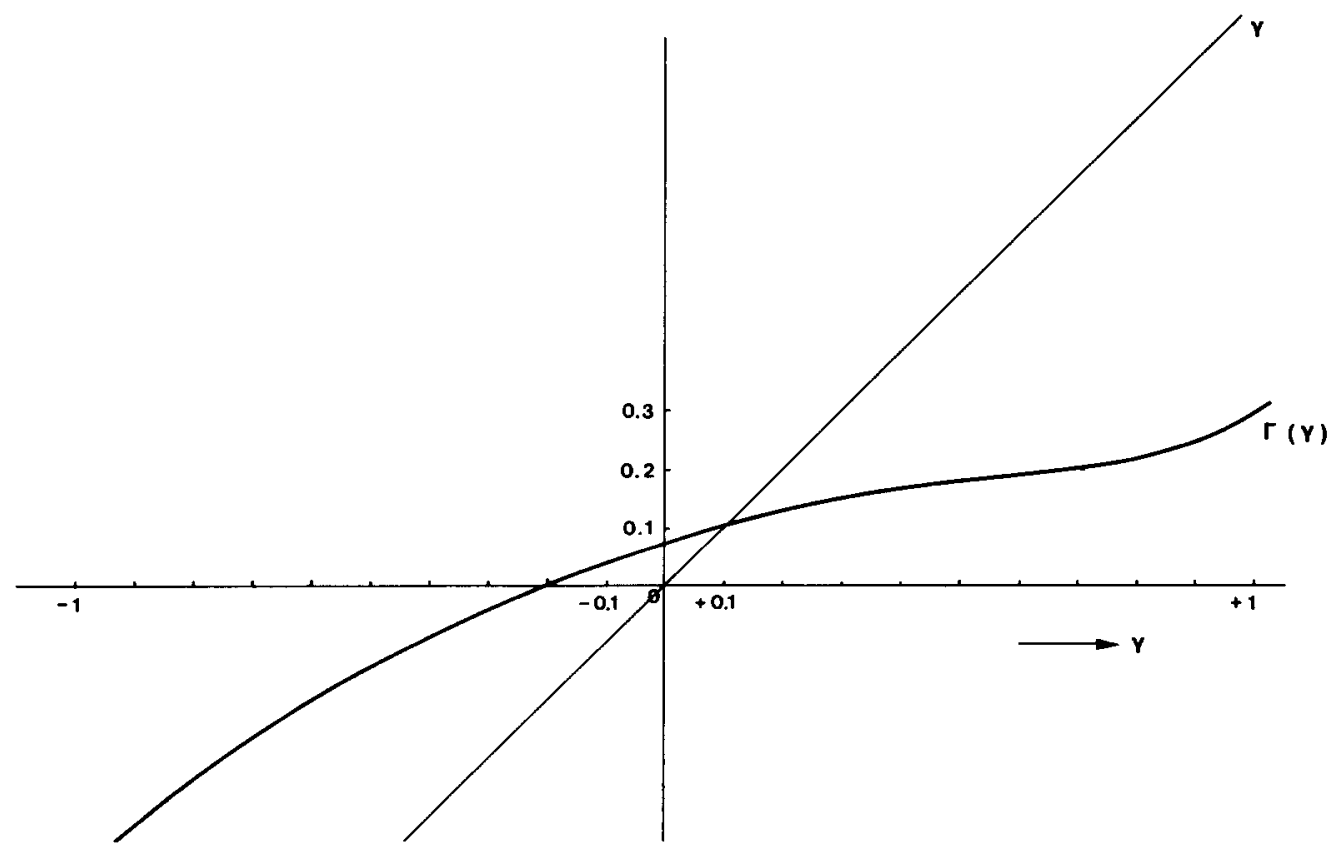

Fig. 7. The function $\Gamma(\gamma)[18,21]$. 
Table 10

\begin{tabular}{cc|lc}
\multicolumn{4}{c}{$\epsilon$ as a function of $\gamma[18,21]$} \\
\hline$\gamma$ & $\epsilon$ & $\gamma$ & $\epsilon$ \\
\hline-1 & -2.8281 & 0.1 & -1.7394 \\
-0.9 & -2.7010 & 0.2 & -1.6752 \\
-0.8 & -2.5798 & 0.3 & -1.6185 \\
-0.7 & -2.4644 & 0.4 & -1.5705 \\
-0.6 & -2.3545 & 0.5 & -1.5337 \\
-0.5 & -2.2501 & 0.6 & -1.5129 \\
-0.4 & -2.1511 & 0.65 & -1.5116 \\
-0.3 & -2.0575 & 0.7 & -1.5196 \\
-0.2 & -1.9694 & 0.8 & -1.5875 \\
-0.1 & -1.8868 & 0.9 & -1.8856 \\
0 & -1.8101 & 0.95 & -2.5432 \\
\hline
\end{tabular}

3. Examples. Ising and isotropic XY systems with a transverse magnetic field (Jullien, Pfeuty, Fields and Doniach [15], Jullien and Pfeuty [16], Penson, Jullien and Pfeuty [17])

The examples of this section are systems of spins $\frac{1}{2}$ in an external magnetic field, which gives an extra term in the Hamiltonian, linear in this field $h$. The ground-state energy of these systems may also be approximated with the aid of RG techniques. The corresponding formalism has been developed by Jullien et al. $[15,16,17]$.

Again the starting point is the formulation of a spin Hamiltonian

$$
H\left(S_{i} ; \gamma, h\right)=-4 J \sum_{\langle i, j\rangle}\left[\frac{1}{2}(1+\gamma) S_{i, x} S_{j, x}+\frac{1}{2}(1-\gamma) S_{i, y} S_{j, y}\right]-2 h \sum_{i} S_{i, z}, \quad J>0 .
$$

This Hamiltonian takes the place of (1), the latter representing the interactions in systems without external fields.

The spin interaction in (9) is between nearest-neighbour pairs $\langle i, j\rangle$ and two special cases will be discussed, the Ising systems $(\gamma=1)$ and the isotropic XY systems $(\gamma=0)$. For the external field a suitable unit is chosen, such that the corresponding term in $H$ has the form: $-2 h \Sigma_{i} S_{i, z}$. In contradistinction to the literature cited here $[15,16,17]$, the spin components are represented by $\frac{1}{2}$ times the Pauli matrices, a convention used throughout this paper. The spins in (9) are sited on the points $i$ of a $d$-dimensional lattice; in the work of Jullien et al. (l.c.) restriction is made to $d=1$ and 2 .

Again the system is divided into cells, not necessarily of an odd number of spins in this case. Per cell the lowest pair of states corresponding with the internal energy is taken into consideration. This internal energy has two parts, the coupling between spins in the cell and the interaction with the external field $h$. The lowest pair of states is, in general, not a Kramers doublet for two reasons: There is an external magnetic field $h \neq 0$ and the number of spins need not be odd. Projection of $H$ on the product space corresponding with the lowest pair of states per cell gives a similar Hamiltonian, in terms of cell spins $\boldsymbol{S}_{\boldsymbol{k}}^{(1)}, \boldsymbol{k}$ denoting here the centre of the cell. The only difference in form between the two Hamiltonians is an additive constant appearing in the latter. The distance in energy between the lowest two states per cell is expressed in terms of the coupling between the $z$ component of the $S_{k}^{(1)}$ and a renormalized field $h^{(1)}$. This projection, or scaling, is analogous to the one that results in (5). This projection may be repeated leading to a Hamiltonian $H^{(t)}$ after $t$ scaling operations. The Hamiltonian $H^{(t)}$ for the Ising model $(\gamma=1)$ takes the form 


$$
\begin{aligned}
& H^{(t)}=-4 J^{(t)} \sum_{\langle i, j\rangle} S_{i, x}^{(1)} S_{j, x}^{(1)}-2 h^{(t)} \sum_{i} S_{i, z}^{(t)}+N^{(t)} C^{(t)} I^{(t)}, \\
& I^{(t)}=\prod_{i} I_{i}^{(t)}
\end{aligned}
$$

In this formula $S_{i}^{(t)}$ and $I_{i}^{(t)}$ respectively are the spin vector and the two-dimensional unit matrix for the $i$ th spin, which represents a system with two states, i.e. the lowest pair of states of the (isolated) $i$ th cell in the spin lattice that results after $(t-1)$ scaling operations. The analogous Hamiltonian $H^{(t)}$ for the isotropic XY model $(\gamma=0)$ is given by

$$
\begin{aligned}
& H^{(t)}=-2 J^{(t)} \sum_{i, j\rangle}\left(S_{i, x}^{(t)} S_{j, x}^{(t)}+S_{i, y}^{(t)} S_{j, y}^{(t)}\right)-2 h^{(t)} \sum_{i} S_{i, z}^{(t)}+N^{(t)} C^{(t)} I^{(t)}, \\
& I^{(t)}=\prod_{i} I_{i}^{(t)} .
\end{aligned}
$$

In both Hamiltonians (10) and (11) the double sum $\Sigma_{\langle i, j\rangle}$ is over nearest neighbour pairs only, and $J^{(t)}$ and $h^{(t)}$ are renormalized coupling constants. In the additive constant operator: $N^{(t)} C^{(t)} I^{(t)}, N^{(t)}$ is the number of spins $S_{i}^{(t)}$ and it has the value $N / n^{t}, N$ being the original number of spins and $n$ the number of spins per cell. In the discussion of the scaling in more detail, for the two cases $\gamma=1$ and $\gamma=0$ separately, it becomes clear that the interaction between neighbouring cells, in the restriction to the lowest doublets, does not have diagonal matrix elements. This, together with the commutator properties of the components of the $\boldsymbol{S}_{i}^{(t)}$, is the very reason that the symmetry of the coupling between spin pairs is conserved in the scaling operation. The Hamiltonian $H^{(t)}$ for $t=0$ may correspond with the original Hamiltonian $H$, given in (9), with parameters:

$$
J^{(0)}=J, \quad h^{(0)}=h, \quad C^{(0)}=0 .
$$

Now the scaling operation will be considered in more detail, i.e. the derivation of $H^{(t+1)}$ as a projection of $H^{(t)}$. First of all a lattice of cells in the $S_{i}^{(t)}$ lattice is introduced. The cells, being indicated by a vector $\boldsymbol{k}$, give a partition of $H^{(t)}$ into a sum of terms representing internal interactions within the cells and a sum of coupling terms for two cells, this apart from the constant terms in (10) and (11). Both expressions may so be rewritten in the form

$$
H^{(t)}=\sum_{k} H_{k}^{(t)}+\sum_{\langle k, l\rangle} H_{k, l}^{(t)}+N^{(t)} C^{(t)} I^{(t)}
$$

in which the second term is a sum over neighbour pairs of cells. This restriction to neighbour cells immediately follows from the restriction in (10) and (11) to neighbour spin interactions. The restriction to neighbour cells in (13), however, gives a restriction in $H^{(t+1)}$ to neighbour spins. So one may conclude that the range of the interactions is conserved in the scaling operation. The expression (13) is a generalization of formula (2) of the Introduction. Now the eigenvalue problem of the isolated cells represented by $H_{k}^{(t)}$ should be solved exactly in the space of $2^{n}$ states per block. This eigenvalue problem for the Ising model and that for the isotropic XY model have to be discussed separately. A common feature of both problems, however, is that a complete set of states per cell is represented by the direct product of eigenstates of the spin components $S_{i, z}^{(t)}, i$ referring to the spins of a particular cell. It is also true for both systems that the 
eigenstates of $H_{k}^{(t)}$ are linear combinations of states with an even or with an odd number of eigenvalues: $-\frac{1}{2}$ for the individual $S_{i, z}^{(t)}$. This follows from the form of the pair interaction in (9). A state with an even number of eigenvalues: $-\frac{1}{2}$ is called a state with even parity, the others have odd parity. So the eigenstates of $H_{k}^{(t)}$ have a definite parity. For the Ising systems, $\gamma=1$, the lowest pair of levels for an isolated cell $k$ correspond with state vectors: $|+, k\rangle^{(t+1)}$ and $|-, k\rangle^{(t+1)}$. It is stated here without proof that they have different parity $[15,17]$. The energy eigenvalues respectively are: $E_{+}^{(t+1)}$ and $E_{-}^{(t+1)}$. Now a projection operator $P_{0}$ is introduced that projects on the lowest pair of states per cell i.e. the states: $|+, k\rangle^{(t+1)}$ and $|-, k\rangle^{(t+1)}$ for cell $\boldsymbol{k}$. This projection operator is the counterpart of the operator introduced in (3.1) for systems without external field. Operators for the lowest pair of states of cell $\boldsymbol{k}$ may be expressed in terms of a cell spin operator $\boldsymbol{S}_{\boldsymbol{k}}^{(t+1)}$, the $z$ component of which is chosen to be diagonal. The two states may be connected by operators that can be written in terms of the $x$ - or $y$-component of the cell spins. All three components of the $S_{k}^{(t+1)}$ are represented by $\frac{1}{2}$ times the well known Pauli matrices. The coupling terms $-4 J^{(t)} S_{i, x}^{(t)} S_{j, x}^{(t)}$ of $H_{k, l}^{(t)}$, in which the $i$ th spin belongs to cell $k$ and $j$ th spin to cell $l$, only have elements that are non diagonal for both cells, because $S_{i, x}^{(t)}$ as well as $S_{j, x}^{(t)}$ connect states of different parity. Because of the fact that the Hamiltonian $H_{k}^{(t)}$ only contains real terms the eigenstates may be chosen to be real and consequently also the matrix elements of $S_{i, x}^{(t)}$ and $S_{j, x}^{(t)}$. So the projection of the $x$ and $z$ components of the spins $S_{i}^{(t)}$ of cell $k$ on the corresponding lowest doublet may be written

$$
\begin{aligned}
& P_{0} S_{i, z}^{(t)} P_{0}=\frac{1}{2}\left[{ }^{(t+1)}\left\langle+, k\left|S_{i, z}^{(t)}\right|+, k\right\rangle^{(t+1)}+{ }^{(t+1)}\left\langle-, k\left|S_{i, z}^{(t)}\right|-, k\right\rangle^{(t+1)}\right] I^{(t+1)}+\zeta_{i}^{(t)} S_{k, z}^{(t+1)} \\
& P_{0} S_{i, x}^{(t)} P_{0}=\xi_{i}^{(t)} S_{k, x}^{(t+1)}
\end{aligned}
$$

in which $S_{k, z}^{(t+1)}$ and $S_{k, x}^{(t+1)}$ are components of a cell spin. The results of the projection of the different terms in (13) for the Ising systems may now be summarized (cf. Jullien, Pfeuty, Fields and Doniach [15], Penson, Jullien and Pfeuty [17]):

$$
\begin{aligned}
& P_{0} H_{k}^{(t)} P_{0}=-2 h^{(t+1)} S_{k, z}^{(t+1)}+\frac{1}{2}\left(E_{+}^{(t+1)}+E_{-}^{(t+1)}\right) I^{(t+1)}, \quad h^{(t+1)}=\frac{1}{2}\left(E_{-}^{(t+1)}-E_{+}^{(t+1)}\right), \\
& P_{0} H_{k, l}^{(t)} P_{0}=-4 J^{(t+1)} S_{k, x}^{(t+1)} S_{i, x}^{(t+1)}
\end{aligned}
$$

The choice is made that the state for which $S_{k, z}^{(t+1)}$ has the eigenvalue $+\frac{1}{2}$, always represents the state with even parity, indicated by the + sign. For reasons of symmetry the coefficients $J^{(t+1)}$ are the same for all neighbour pairs of cells. The constants $\xi_{i}, \zeta_{i}$, however, may depend on the index $i$, i.e. on the position of the spin in the Kadanoff cell. A sum of products of coefficients $\xi_{i}$ for neighbour spin pairs of different cells determine the coefficient $J^{(t+1)}$.

The formulas (15) give sufficient information to determine also the renormalized additive part of the Hamiltonian per cell: $C^{(t+1)}$. This constant obeys the recurrence relation

$$
C^{(t+1)}=n C^{(t)}+\frac{1}{2}\left(E_{+}^{(t+1)}+E_{-}^{(t+1)}\right)
$$

as follows from (13) and the first line of (15).

Now the formulas (15) and (16) give the general recipe for the determination of the renormalized coefficients $J^{(t+1)}, h^{(t+1)}$ and $C^{(t+1)}$ of the Hamiltonian $H^{(t+1)}$, in terms of the parameters of $H^{(t)}$, defined in (10). So the RG transformation may be carried out and an upper bound for the ground-state energy per spin may be found 


$$
\lim _{N \rightarrow \infty} \frac{E_{0}}{N}=\lim _{t \rightarrow \infty} \frac{C^{(t)}}{n^{t}}=\frac{1}{2} \sum_{s=1}^{\infty} \frac{E_{+}^{(s)}+E_{-}^{(s)}}{n^{s}}
$$

as a generalization of formula (7) of the Introduction. Again it can be shown that the nonconstant part of (10) does not contribute to (17) in the limit $t \rightarrow \infty$. As is discussed in the work of Jullien et al. [15], the ratio of the parameters $h^{(t)} / J^{(t)}$ converges to a limit value for $t \rightarrow \infty$. For $h / J=h^{(0)} / J^{(0)}<(h / J)_{\mathrm{C}},(h / J)_{\mathrm{C}}$ being an unstable fixed point, the limit value equals: 0 , whereas for $h / J>(h / J)_{\mathrm{C}}$ this limit value is: $+\infty$. For linear systems the fixed point $(h / J)_{C}$ tends to the value 1 for $n \rightarrow \infty$. This limit value represents a singular point for the ground-state energy per spin $\epsilon=\lim _{N \rightarrow \infty} E_{0} / N$ as a function of $h / J$ (see fig. 2 of ref. [15]). The second derivative: $-\mathrm{d}^{2} \epsilon / \mathrm{d} h^{2}$ diverges logarithmically for $h / J=1$, and it can be proven that there is a strict equivalence of this singularity to the critical behavior of the Ising model in two dimensions, close to $T_{\mathrm{C}}$. Especially close to singular points the RG method is well-adapted to calculate values of $\epsilon(h / J)$.

Now an outline will be given of the derivation of an expression analogous to (17), for the isotropic XY systems. For these systems the eigenstates of the $H_{k}^{(t)}$ may be classified according to the eigenvalues of $\Sigma_{i(k)} S_{i, z}^{(t)}$ the sum being restricted to the $k$ th cell. These eigenvalues may be written: $\frac{1}{2}(-n+2 q-2)$, $q=1, \ldots, n+1$, as before $n$ denoting the number of spins per cell. The eigenvalues of the $H_{k}^{(t)}$ are linear functions of $h^{(t)}$, the corresponding $q$ being a good quantum number.

The lowest eigenvalue for a fixed $q$, may be expressed in the form

$$
E_{q}^{(t+1)}=(n-2 q+2) h^{(t)}+e_{q} J^{(t)}
$$

the constant $e_{q}$ being independent of the value of $h^{(t)}$. It can be proven that the lowest pair of states correspond with adjacent quantum numbers, $q$ and $q+1$. The corresponding state vectors for cell $k$ may be denoted by: $|q, \boldsymbol{k}\rangle^{(t+1)}$ and $|q+1, \boldsymbol{k}\rangle^{(\boldsymbol{t}+1)}$ respectively. For sufficiently large negative values of $h, q=1$ for all $t$ and for sufficiently large positive values, $q=n$, also for all $t[16]$.

Again the total Hamiltonian (11) will be projected on the product space of the sets $\left\{|q, k\rangle^{(t+1)}\right.$, $\left.|q+1, \boldsymbol{k}\rangle^{(t+1)}\right\}$. In the representation of this projection, again use can be made of the spin vectors $\boldsymbol{S}_{k}^{(t+1)}$. The state $q+1$ will have quantum number $+\frac{1}{2}$ for $S_{k, z}^{(t+1)}$ and the state $q$ the quantum number $-\frac{1}{2}$. So one finds for the projections of the $H_{k}^{(t)}$ expressions analogous to those given in the first line of (15) (cf. also ref. [16])

$$
\begin{aligned}
& P_{0} H_{k}^{(t)} P_{0}=-2 h^{(t+1)} S_{k, z}^{(t+1)}+\frac{1}{2}\left(E_{q}^{(t+1)}+E_{q+1}^{(t+1)}\right) I^{(t+1)}, \\
& h^{(t+1)}=\frac{1}{2}\left(E_{q}^{(t+1)}-E_{q+1}^{(t+1)}\right)=h^{(t)}+\frac{1}{2}\left(e_{q}-e_{q+1}\right) J^{(t)} .
\end{aligned}
$$

The two lowest states per cell, also for the XY systems, have different parity and may, in principle, be connected by the terms $H_{k, l}^{(t)}$ in (13), which are bilinear in $x$ - and $y$-components of spins of the adjacent cells $k$ and $l$. The matrix elements of the different $S_{i, x}^{(t)}$ and $S_{i, y}^{(t)}, i$ belonging to cell $k$, may again be represented by Pauli matrices. First of all one may write down the well-known commutator properties of the spins $S_{i}^{(t)}$

$$
\left[S_{i, z}^{(t)}, S_{j, x}^{(t)}\right]=\mathrm{i} \delta_{i, j} S_{i, y}^{(t)}, \quad\left[S_{i, z}^{(t)}, S_{j, y}^{(t)}\right]=-\mathrm{i} \delta_{i, j} S_{i, x}^{(t)}
$$

from which the following relations in the manifold $\left\{|q, k\rangle^{(t+1)},|q+1, k\rangle^{(t+1)}\right\}$ may be derived 


$$
\begin{aligned}
& { }^{(t+1)}\left\langle q, k\left|S_{i, x}^{(t)}\right| q+1, k\right\rangle^{(t+1)}=\mathrm{i}^{(t+1)}\left\langle q, k\left|\left[S_{i, z}^{(t)}, S_{i, y}^{(t)}\right]\right| q+1, k\right\rangle^{(t+1)} \\
& =\mathrm{i}^{(t+1)}\left\langle q, k\left|\left[\sum_{j(k)} S_{j, z}^{(t)}, S_{i, y}^{(t)}\right]\right| q+1, k\right\rangle^{(t+1)} \\
& =\mathrm{i}\left[{ }^{(t+1)}\left\langle q, \boldsymbol{k}\left|\sum_{j(\boldsymbol{k})} S_{j, z}^{(t)}\right| q, \boldsymbol{k}\right\rangle^{(t+1)}-{ }^{(t+1)}\left\langle q+1, \boldsymbol{k}\left|\sum_{j(k)} S_{j, z}^{(t)}\right| q+1, \boldsymbol{k}\right\rangle^{(t+1)}\right]{ }^{(t+1)}\left\langle q, \boldsymbol{k}\left|S_{i, y}^{(t)}\right| q+1, \boldsymbol{k}\right\rangle^{(t+1)} \\
& =-\mathrm{i}^{(t+1)}\left\langle q, \boldsymbol{k}\left|\boldsymbol{S}_{i, y}^{(t)}\right| q+1, \boldsymbol{k}\right\rangle^{(t+1)} \text {. }
\end{aligned}
$$

If one takes into consideration that the $q$ state is represented by the eigenvalue: $-\frac{1}{2}$ of $S_{k, z}^{(t+1)}$ and the $q+1$ state by the eigenvalue: $+\frac{1}{2}$, the projection of all components $S_{i, x}^{(t)}, S_{i, y}^{(t)}$ and $S_{i, z}^{(t)}$ of spin $i$ may be represented by the components of a Pauli spin, apart from additive and multiplicative constants, as in (14)

$$
\begin{aligned}
& P_{0} S_{i, z}^{(t)} P_{0}=\frac{1}{2}\left[{ }^{(t+1)}\left(q, k\left|S_{i, z}^{(t)}\right| q, k\right\rangle^{(t+1)}+{ }^{(t+1)}\left\langle q+1, k\left|S_{i, z}^{(t)}\right| q+1, k\right\rangle^{(t+1)}\right] I^{(t+1)}+\zeta_{i}^{(t)} S_{k, z}^{(t+1)} \\
& P_{0} S_{i, x}^{(t)} P_{0}=\xi_{i}^{(t)} S_{k, x}^{(t+1)}, \quad P_{0} S_{i, y}^{(t)} P_{0}=\xi_{i}^{(t)} S_{k, y}^{(t+1)}
\end{aligned}
$$

In these formulas the cylindrical symmetry of the projection is apparent in the same coefficient $\xi_{i}^{(t)}$ for the $x$-and the $y$-component of spin $i$. So the cylindrical symmetry is conserved in the projection. For the evaluation of $H^{(t+1)}$ one has to know the projection of the $H_{k}^{(t)}$, already given in (19), as well as the projection of the interaction terms $H_{k, l}^{(i)}$, which may be derived in a particular example on the basis of (21). The general result has the form [16]

$$
P_{0} H_{k, l}^{(t)} P_{0}=-2 J^{(t+1)}\left(S_{k, x}^{(t+1)} S_{l, x}^{(t+1)}+S_{k, y}^{(t+1)} S_{l, y}^{(t+1)}\right),
$$

in which the renormalized coupling constant is a linear function of $J^{(t)}$. The actual calculation of the renormalized constants $h^{(t+1)}$ and $J^{(t+1)}$ has to be performed for the XY systems of different dimension separately. Here only the overall symmetry of the scaled Hamiltonian $H^{(t+1)}$, as a consequence of the same symmetry of $H^{(t)}$, is derived. Now the renormalized constant part of Hamiltonian $H^{(t+1)}$ may be found combining the results in (19) and (22)

$$
\begin{aligned}
C^{(t+1)} & =n C^{(t)}+\frac{1}{2}\left(E_{q}^{(t+1)}+E_{q+1}^{(t+1)}\right) \\
& =n C^{(t)}+(n-2 q+1) h^{(t)}+\frac{1}{2}\left(e_{q}+e_{q+1}\right) J^{(t)}
\end{aligned}
$$

and the corresponding upper bound for the ground-state energy per spin in the XY systems is given by

$$
\lim _{N \rightarrow \infty} \frac{E_{0}}{N}=\lim _{t \rightarrow \infty} \frac{C^{(t)}}{n^{t}}=\frac{1}{2} \sum_{s=1}^{\infty} \frac{E_{q}^{(s)}+E_{q+1}^{(s)}}{n^{s}}
$$

at least for those cases in which $q$, representing the lowest two states per cell, is not changed in the RG transformation. This condition is fulfilled for values of $h$ that are sufficiently large positive or negative, i.e. $q=n$ and $q=1$ respectively. The stable fixed points for these two cases are given by $h / J=$ $+\infty(q=n)$ and $h / J=-\infty(q=1)$. Also for the XY model there is a parallelism between the expression for the upper bound of the lowest energy eigenvalue (24), and the corresponding expression (7) for Heisenberg systems without external field. 
Detailed numerical calculations for the systems with external fields are not given in this paper. For the $\mathrm{XY}$ system $(d=1)$ these are given in ref. [16], together with a comparison with the exact values in the literature.

\section{Concluding remarks}

(1) First of all a comparison could be made between the different values for $\epsilon(0)$ for the linear chain, i.e. the ground-state energy per spin of the Heisenberg chain, with only nearest-neighbour interactions.

The approximation methods of this paper give three different values, i.e. those of the subsections 2.1 , 2.4 and 2.5 , which respectively are

$$
\epsilon_{0}^{*}(0)=-1.5960, \quad \epsilon^{*}(0)=-1.56522, \quad \epsilon(0)=-1.8101 \text {. }
$$

These results may be compared with Hulthén's exact value [24], denoted by $\epsilon_{\mathrm{H}}(0)$, and the exact value for a finite chain of 10 spins, computed by Majumdar and Ghosh [42], $\epsilon_{\mathrm{MG}}(0)$. The results of the last two authors for $|\gamma|<1$, are also drawn in fig. 1. For $\gamma=0$ one so has the following values for comparison

$$
\epsilon_{\mathrm{H}}(0)=1-4 \log 2=-1.7726, \quad \epsilon_{\mathrm{MG}}(0)=-1.8062 \text {. }
$$

As was known already $\epsilon_{0}^{*}(0)$ and $\epsilon^{*}(0)$ give an upper bound for the energy per spin for the infinite chain. It turns out that $\epsilon(0)$ is an underestimate that only differs about $2 \%$ from the exact value.

The first-order expression $\epsilon_{0}^{*}(\gamma)$ of (1-7) is also an upper bound for $\gamma \neq 0$. It may be compared with Niemeijer's result [43], which also gives a linear function of $\gamma$

$$
\epsilon_{\mathrm{N}}(\gamma)=4\left(-1 / \pi-1 / \pi^{2}+2 \gamma / \pi^{2}\right)=-1.6785+0.8106 \gamma
$$

This upper bound is a sharper one for $\gamma \leq 0.4$. It is also drawn in fig. 1 . For $\gamma \geq 0.5$, however, the best upper bound is given by $\epsilon^{*}(\gamma)$ of subsection 2.4 .

(2) The division of the lattice into Kadanoff cells, in the case of one single Bravais lattice, gives the possibility to determine also a lower bound for the energy per spin in the ground state. The interactions should be restricted to nearest neighbours.

Examples of these spin lattices are the Heisenberg chain and the square and cubic lattices with Heisenberg interactions between nearest neighbours only.

Finite systems with periodic boundary conditions are considered here, in the limit of $N \rightarrow \infty, N$ being the total number of spins. The hypothesis is made that in the ground state every pair of neighbouring spins contributes the same amount to the total energy.

The partition of the total Hamiltonian $H$, corresponding with the division of the lattice into Kadanoff cells: $H=H_{0}+H^{\prime}$, was discussed at length in section 1 . The cells are rows of an odd number of consecutive spins, squares or cubes, also with an odd number of spins, respectively in the one-, two- and three-dimensional examples given above.

For an isolated cell the number of interactions between spin pairs equals

$$
(2 l+1)^{d} d-\frac{1}{2}(2 l+1)^{d-1} \cdot 2 d=(2 l+1)^{d-1} \cdot 2 l,
$$

in which expression $d$ denotes the dimension of the lattice and $(2 l+1)^{d}, l=1,2,3, \ldots$ is the number of 
spins per cell, which was chosen to be odd in section 1. From simple geometrical considerations it follows that the bonds between the cells contribute the fraction $1 /(2 l+1)$ to the total energy of the ground state.

Now the lowest eigenvalue of $H$ may be written as the sum of the expectation values of $H_{0}$ and $H^{\prime}$ in the ground state. For the first expectation value one has a lower bound, represented by its lowest eigenvalue. Asymptotically it will hold

$$
\langle H\rangle=N \epsilon+o(N)=\left\langle H_{0}\right\rangle+\left\langle H^{\prime}\right\rangle \geq \frac{N e_{0}}{(2 l+1)^{d}}+\frac{N \epsilon}{2 l+1}+o(N),
$$

in which expression $\epsilon$ is the exact ground-state energy per spin for $N \rightarrow \infty$ and $e_{0}$ the lowest eigenvalue of $H_{0}$ per cell. The symbol o $(N)$ denotes a term of vanishing order as compared to $N$, for $N \rightarrow \infty$.

Now a lower bound for $\epsilon$ in terms of $e_{0}$ immediately follows from the inequality given above

$$
\epsilon \geq \frac{2 l+1 \frac{e_{0}}{2 l(2 l+1)^{d}}}{\underline{2 l}}
$$

For the Heisenberg chain $(d=1)$ with cells of three spins $(l=1) e_{0}$ is given in table $7: e_{0}=-4(\gamma=0)$, and a lower bound for $\epsilon$ is found to be: -2 . This is a mere illustration of the method. A better value can be found by taking larger cells, e.g. $l=3$, for which the corresponding eigenvalue $e_{0}$ equals: -11.3450 . This gives the lower bound

$$
\epsilon \geq-\frac{1}{6} \cdot 11.3450=-1.891 \text {, }
$$

which differs $\approx 7 \%$ from the exact value by Hulthén [24].

(3) So far the results of the renormalization techniques for higher dimensions have been restricted to a few examples, the one given in subsection 2.3 being the most interesting application. In that case, the triangular lattice, the numerical outcome of the calculations fairly agrees with other results in the literature, but has the additional advantage of being an upper bound.

For the square and cubic lattices also interesting results may be expected, especially along the lines of the methods of subsections 2.4 and 2.5. To master the computational difficulties for these examples additional approximations are necessary and the lengthy numerical calculations should be made tractable with modern computer facilities.

\section{Acknowledgements}

The author is very much indebted to Dr. F.W. Wiegel and Dr. M.W.M. Willemse for critical reading of the manuscript and for several remarks, which enabled him to improve his text.

\section{Appendix A: Algebraic properties of the spin [7,23]}

The spin is an intrinsic property of elementary particles. It is an example of an angular momentum vector $J$, with components $J_{x}, J_{y}, J_{z}$ in a given rectangular frame. 
If considered as quantum-mechanical variables the components obey the well-known commutation relations

$$
\left[J_{x}, J_{y}\right]=\mathrm{i} J_{z}, \quad\left[J_{y}, J_{z}\right]=\mathrm{i} J_{x}, \quad\left[J_{z}, J_{x}\right]=\mathrm{i} J_{y}, \quad \hbar=1
$$

A complete set of irreducible matrix representations is given by

$$
\begin{aligned}
& \left(j m+1\left|J_{x}\right| j m\right)=\frac{1}{2}[(j-m)(j+m+1)]^{1 / 2}, \\
& \left(j m-1\left|J_{x}\right| j m\right)=\frac{1}{2}[(j+m)(j-m+1)]^{1 / 2}, \\
& \left(j m+1\left|J_{y}\right| j m\right)=-\frac{i}{2}[(j-m)(j+m+1)]^{1 / 2}, \\
& \left(j m-1\left|J_{y}\right| j m\right)=\frac{i}{2}[(j+m)(j-m+1)]^{1 / 2}, \\
& \left(j m\left|J_{z}\right| j m\right)=m, \quad j=\frac{1}{2}, 1, \frac{3}{2}, 2, \ldots, \quad m=-j,-j+1, \ldots, j-1, j .
\end{aligned}
$$

A representation is characterized by the quantum number $j$. It corresponds with a set of $(2 j+1)$ dimensional square matrices, rows and columns of which are numbered by the quantum numbers $m^{\prime}$ and $m$ in the expressions $\left(j m^{\prime}\left|J_{x}\right| j m\right)$ etc. etc. For $J_{x}$ and $J_{y}$ one has the selection rule $m^{\prime}=m \pm 1$, whereas for $J_{z}: m^{\prime}=m$. All matrix elements not written down in (A.2) are zero.

For an intrinsic angular momentum the quantum number $j$ has a fixed value, and consequently such a momentum is completely described by one single representation. This in contradistinction to e.g. the orbital angular momentum $L$ of atoms for which the quantum number $j$, most times called $L$ in this case, may have any of the values $0,1,2, \ldots$

Half-integral values of $j$ are restricted to those systems that contain an odd number of elementary particles of half-integral spin.

In this work only particles with intrinsic spin moment $\frac{1}{2}$ and without any other angular momentum are considered. For the representation of this spin moment $S$ the so called Pauli matrices $\sigma_{x}, \sigma_{y}$ and $\sigma_{z}$ are used

$$
\boldsymbol{S}=\frac{1}{2} \boldsymbol{\sigma}, \quad \sigma_{x}=\left[\begin{array}{ll}
0 & 1 \\
1 & 0
\end{array}\right], \quad \sigma_{y}=\left[\begin{array}{rr}
0 & -\mathrm{i} \\
\mathrm{i} & 0
\end{array}\right], \quad \sigma_{z}=\left[\begin{array}{rr}
1 & 0 \\
0 & -1
\end{array}\right] .
$$

It is immediately seen that (A.3) is an example $\left(j=\frac{1}{2}\right)$ of the general form of the representations given in (A.2). A complete set of states or spinors corresponding with the operators (A.3) is given by the column vectors

$$
\alpha=\left(\begin{array}{l}
1 \\
0
\end{array}\right), \quad \beta=\left(\begin{array}{l}
0 \\
1
\end{array}\right)
$$

which are eigenstates of $S_{z}\left(\sigma_{z}\right)$ with eigenvalues: $\pm \frac{1}{2}( \pm 1)$.

The set (A.3) in combination with the unit matrix

$$
I=\left[\begin{array}{ll}
1 & 0 \\
0 & 1
\end{array}\right],
$$

is a complete set of self-adjoint two-dimensional matrices. 
Systems of $N$ spins $\frac{1}{2}$, discussed in this work, have physical variables that may be written as direct products of $N$ factors of the type (A.3) or (A.5).

The components of the $i$ th spin e.g., are given by

$$
S_{i}=I \otimes I \ldots \otimes S \otimes \ldots \otimes I \otimes I,
$$

the symbols $\otimes$ denoting a repeated direct product. The $i$ th factor in (A.6) is represented by the matrices $S_{x}, S_{y}$ and $S_{z}$, given in (A.3). Two-spin variables like $S_{i z} S_{j x}$ are represented by

$$
S_{i z} S_{j x}=I \otimes I \otimes \ldots \ldots \otimes S_{z} \otimes \ldots \otimes S_{x} \otimes \ldots \otimes I \otimes I,
$$

or by linear combinations of such forms. The factors $S_{z}$ and $S_{x}$ in (A.7) are on the $i$ th and $j$ th position respectively. The exchange interaction, as an example, may be written for the pair $(i, j)$, as a linear combination of expressions (A.7) if one writes

$$
J\left(S_{i} \cdot S_{j}\right)=J\left(S_{i x} S_{j x}+S_{i y} S_{j y}+S_{i z} S_{j z}\right) .
$$

The space of spinor states is the direct product of the spaces represented by (A.4) and conveniently written as

$$
\begin{aligned}
& \alpha_{1} \otimes \alpha_{2} \otimes \ldots \otimes \alpha_{N} \\
& \beta_{1} \otimes \alpha_{2} \otimes \ldots \otimes \alpha_{N} \\
& \alpha_{1} \otimes \beta_{2} \otimes \ldots \otimes \alpha_{N}, \text { etc. etc. }
\end{aligned}
$$

The total number of states equals $2^{N}$.

The renormalization techniques developed in this work are essentially projection techniques in the space (A.8). The projection is on a space of product states like (A.8), the products having a number of factors being the fraction $1 / n$ of the original number $N$, each factor corresponding with one cell of $n$ spins.

Each factor represents a cell spin $\frac{1}{2}$ having algebraic properties that are isomorphous with those of the original spins. This is written out in formula (4) of the Introduction. This isomorphism is a consequence of Kramers' theorem discussed in appendix B.

\section{Appendix B: Kramer's theorem [44]}

Kramers' theorem states that all energy levels of a system containing an odd number of electrons must be at least doubly degenerate, provided that there are no magnetic fields acting on the system.

This theorem is a consequence of the requirement that the equations of motion are invariant for time-reversal or time-inversion. Classically time-reversal simply is inversion of all velocities, which results in a retracing of all particles of their paths.

The quantum analogue is formulated in terms of a time-reversal operator $T$ acting on the wave function $\psi$. For a Hamilton $H$ without spin variables $T$ simply transforms $\psi$ into $\psi^{*}$, which immediately follows from the time-dependent Schrödinger equation 
$H \psi=\mathrm{i} \hbar \partial \psi / \partial t$

For a real Hamiltonian complex conjugation results in

$$
H \psi^{*}=\mathrm{i} \hbar \partial \psi^{*} / \partial(-t)
$$

which formula shows that $\psi^{*}$ is the solution of the mechanical problem for time flowing backwards. Complex conjugation of $\psi$ does not affect $|\psi|^{2}$, the probability density, whereas the momentum distribution for a single electron $k$ proportional to the imaginary part of $\psi^{*} \nabla_{k} \psi$ is inverted, as it should be. Energy is conserved because $H \psi=E \psi$ results in $H \psi^{*}=E \psi^{*}$.

External magnetic fields present should be inverted in the time-reversal operation because the coupling with the magnetic moments should be invariant. These magnetic moments are coupled with electron motion also being inverted in the operation. The time inversion operator is antilinear and antiunitary because

$$
\begin{aligned}
& T(c \psi)=c^{*} T \psi, \\
& |(T \psi, T \phi)|=|(\psi, \phi)|
\end{aligned}
$$

In (B.4) the symbol $(\psi, \phi)$ denotes the inproduct of two state functions. Taking into account spin time-reversal cannot be represented by simple complex conjugation, because in the usual representation with Pauli matrices (A.3), $S_{x}$ and $S_{z}$ are real operators. The antiunitary operator, now defining time inversion

$$
T=U K, \quad U=\sigma_{1 y} \otimes \sigma_{2 y} \otimes \ldots \otimes \sigma_{n y},
$$

in which $K$ is complex conjugation, has all the required properties. The unitary operator $U$ is the direct product of the $y$-components of the vectors $\sigma$, one for each spin. Whereas the factor $K$ represents time inversion for the ordinary degrees of freedom of the system, each Pauli matrix $\sigma_{k y}$ gives inversion of the components $\sigma_{k x}$ and $\sigma_{k z}$, because of the algebraic properties of the $\sigma$ matrices, represented in (A.3),

$$
\sigma_{y}^{2}=1, \quad \sigma_{y} \sigma_{x} \sigma_{y}=-\sigma_{y}^{2} \sigma_{x}=-\sigma_{x}, \quad \sigma_{y} \sigma_{z} \sigma_{y}=-\sigma_{y}^{2} \sigma_{z}=-\sigma_{z}
$$

The $y$-component of $\sigma_{k}$, however, commutes with $U$, but this operator, being purely imaginary, is transformed in the proper way by $K$,

$$
K \sigma_{y} K=-K^{2} \sigma_{y}=-\sigma_{y} .
$$

From the definition of $T$ given in (B.5) one easily derives its effect on a state function $\psi\left(r_{1}, \mu_{1}, \ldots, r_{n}, \mu_{n}\right)$ with variables $r_{1}, \ldots, r_{n}$, representing spatial co-ordinates of the electrons and $\mu_{k}$ the quantized $z$ components of the spin vectors. Restriction to eigenstates of $\sigma_{k z}$ is not essential, but simplifies the argument. One has

$$
T \psi\left(r_{1}, \mu_{1}, \ldots, r_{n}, \mu_{n}\right)=\mathrm{i}^{2\left(\mu_{1}+\mu_{2} \ldots+\mu_{n}\right)} \psi\left(r_{1},-\mu_{1}, \ldots, r_{n},-\mu_{n}\right)^{*},
$$

from which is follows that 


$$
T^{2}=-1
$$

for an odd number of electrons. The result (B.9), however, implies that $T \psi$ and $\psi$ are orthogonal. This in combination with the fact that $T \psi$ and $\psi$ have the same energy eigenvalue $E$, shows that this eigenvalue should be at least doubly degenerate. Generalization of the argument gives an even fold degeneracy.

The proof of the orthogonality of $T \psi$ and $\psi$ is given by noting first that for any two state functions

$$
(T \psi, T \phi)=(U K \psi, U K \phi)=(K \psi, K \phi)=(\psi, \phi)^{*}=(\phi, \psi)
$$

from which it follows

$$
\begin{aligned}
& (T \psi, \psi)=\left(T \psi, T^{2} \psi\right)=-(T \psi, \psi), \\
& (T \psi, \psi)=0
\end{aligned}
$$

For the case of a system of particles with a spin $\frac{1}{2}$, external magnetic fields should be inverted in the process of time inversion. No fields present, however, time inversion results in the orthogonal state $T \psi$, which demonstrates the Kramers' theorem formulated in the first lines of this appendix.

\section{Appendix C: The Wigner-Eckart theorem $[23,44]$}

In this appendix the formulation of the Wigner-Eckart (WE) theorem and a general outline of its proof are given. In the outline reference is made to the quantum theory of angular momentum [23] and to group theory [44].

The WE theorem formulates relations for the matrix elements of tensor operators in a representation for which the total angular momentum and its $z$-component are good quantum numbers.

The states of the system are represented by

$$
\begin{aligned}
& \left.|\gamma j m\rangle, J^{2} \mid \gamma j m\right)=j(j+1)|\gamma j m\rangle, \\
& \left.J_{z} \mid \gamma j m\right)=m|\gamma j m\rangle, \quad m=-j,-j+1, \ldots,+j, \quad \hbar=1,
\end{aligned}
$$

in which $J=\left(J_{x}, J_{y}, J_{z}\right)$ is the total angular momentum vector. The symbol $\gamma$ stands for all other non-specified quantum numbers. For the systems considered in this paper $\boldsymbol{J}$ equals the total spin vector $\boldsymbol{S}$.

Tensor operators are represented by their components

$$
T(k, q), \quad q=-k, \ldots,+k, \quad k=0,1,2,3 \ldots
$$

which transform under rotations like the well known spherical harmonics $Y_{k q}$. The set $T(k, q)$, like $Y_{k q}$, generates an irreducible representation of the rotation group, which is denoted by $\mathscr{D}_{q^{\prime} q}^{(k)}(\omega)$ in the notation of Tinkham [44].

The properties of $T(k, q)$ may also be formulated in terms of the commutation relations with the components of the total angular momentum, these components being proportional to the operators of 
infinitesimal rotations [23]. The commutation relations are

$$
\begin{aligned}
& {\left[J_{ \pm}, T(k, q)\right]=\sqrt{(k \mp q)(k \pm q+1)} T(k, q \pm 1), \quad J_{ \pm}=J_{x} \pm \mathrm{i} J_{y},} \\
& {\left[J_{z}, T(k, q)\right]=q T(k, q) .}
\end{aligned}
$$

The transformation properties of $T(k, q)$ partly determine the value of their matrix elements for the set of states $|\gamma j m\rangle$ introduced in (C.1). The states $|\gamma j m\rangle$ as well as the operators represent irreducible representations of the rotation group and only for the case that the product representation corresponding with a matrix element is the identical one $(k=0)$, this matrix element is unequal to zero. This may be expressed in a very compact form by introducing so-called reduced matrix elements and the vector-coupling or Clebsch-Gordan coefficients [23], in terms of which the WE theorem takes the form

$$
\left\langle\gamma^{\prime} j^{\prime} m^{\prime}|T(k, q)| \gamma j m\right\rangle=(-)^{\prime}-m^{\prime}\left(\begin{array}{ccc}
j^{\prime} & k & j \\
-m^{\prime} & q & m
\end{array}\right)\left(\gamma^{\prime} j^{\prime}\|\mathbf{T}(k)\| \gamma j\right)
$$

In this expression $\left(\begin{array}{ccc}j^{\prime} & k & j \\ -m^{\prime} & q & m\end{array}\right)$ is a vector-coupling coefficient and $\left(\gamma^{\prime} j^{\prime}\|\mathbf{T}(k)\| \gamma j\right)$ a reduced matrix element. The reduced matrix element is independent of the quantum numbers $m$ and $m^{\prime}$, but may be different for different tensor operators, corresponding e.g. with different parts of the system. All information contained in the transformation properties of the states $|\gamma j m\rangle$ and the operators $T(k, q)$ is expressed in the vector-coupling coefficient in (C.4). The special convention for defining the VCcoefficients used in the literature cited here, [23], leads to the phase factor $(-)^{\prime-m^{\prime}}$.

The quantum numbers $j$ and $j^{\prime}$ are integers or half odd integers according to the total number of spins in the systems. For an even number of spins and with interactions that are rotationally invariant (exchange) $j$ is a good quantum number and has integer values. For an odd number of spins $j$ has half odd values for the same type of interactions. These half odd values are intimately connected with Kramers' degeneracy (appendix B) and the special case $j=S=\frac{1}{2}$ plays a special role in this paper. Formula (1-3.2) is an example of (C.4) with $\gamma^{\prime}=\gamma, j^{\prime}=j=\frac{1}{2}$.

\section{References}

[1] L.P. Kadanoff, Physics 2 (1966) 263.

[2] K.G. Wilson, Phys. Rev. B4 (1971) 3174, 3184.

[3] R. Balescu, Equilibrium and Nonequilibrium Statistical Mechanics (Wiley, 1975) Ch. 10.

[4] S.K. Ma, Modern Theory of Critical Phenomena, Frontiers in Physics, Vol. 46 (Benjamin, 1976) Ch. V.

[5] P. Pfeuty and G. Toulouse, Introduction to the Renormalization Group and to Critical Phenomena, Translated from French by G. Barton (Wiley, 1977) Ch. 4.

[6] E. Lieb and D. Mattis, J. Math. Phys. 3 (1962) 749.

[7] C.J. Thompson, in: Phase Transitions and Critical Phenomena, Vol. I, eds. C. Domb and M.S. Green (Academic Press, London, New York, 1972).

[8] Th. Niemeijer and J.M.J. van Leeuwen, in: Phase Transitions and Critical Phenomena, Vol. VI, eds. C. Domb and M.S. Green (Academic Press, London, New York, 1976) p. 425.

[9] M.H.L. Pryce, Proc. Phys. Soc. (London) A63 (1950) 25.

[10] A. Abragam and M.H.L. Pryce, Proc. Roy. Soc. (London) A205 (1951) 135.

[11] B. Bleany and K.W.H. Stevens, Repts. Progr. Phys. 16 (1953) 108.

[12] W.J. Caspers, Theory of Spin Relaxation (Interscience, 1964) Ch. I.

[13] J.N. Fields, H.W.J. Blöte and J.C. Bonner, J. Appl. Phys. 50 (1979) 1807. 
[14] R. Dekeyser, J. Phys. Suppl. C6 (1978) 747.

[15] R. Jullien, P. Pfeuty, J.N. Fields and S. Doniach, Phys. Rev. B18 (1978) 3568.

[16] R. Jullien and P. Pfeuty, Phys. Rev. B19 (1979) 4646.

[17] K.A. Penson, R. Jullien and P. Pfeuty, Phys. Rev. B19 (1979) 4653.

[18] H.P. van de Braak, W.J. Caspers, C. de Lange and M.W.M. Willemse, Physica 87A (1977) 354.

[19] H.P. van de Braak, W.J. Caspers, F.W. Wiegel and M.W.M. Willemse, J. Stat. Phys. 18 (1978) 577.

[20] H.P. van de Braak, W.J. Caspers and M.W.M. Willemse, Phys. Letters 67A (1978) 147.

[21] H.P. van de Braak, W.J. Caspers, C. de Lange and M.W.M. Willemse, in: Annals of the Israel Physical Society, Vol. 2; Proc. of the 13th IUPAP Conf. on Statistical Physics "Statphys 13", Haifa 1977 (Adam Hilger, Bristol and The Israel Physical Society, in association with The American Institute of Physics, New York, 1978) p. 431.

[22] H.P. van de Braak, W.J. Caspers, P.K.H. Gragert and M.W.M. Willemse, J. Stat. Phys. 20 (1979) 487.

[23] A.R. Edmonds, Angular Momentum in Quantum Mechanics (Princeton University Press, 1960).

[24] L. Hulthén, Arkiv Math. Astron. Fysik 26A (1938) no. 11.

[25] P. Pincus, Solid State Comm. 9 (1971) 1971.

[26] G. Beni and P. Pincus, J. Chem. Phys. 57 (1972) 3531.

[27] J.Y. Dubois and J.B. Carton, J. Phys. (Paris) 35 (1974) 371.

[28] G. Beni, J. Chem. Phys. 58 (1973) 3200.

[29] E. Pytte, Phys. Rev. B10 (1974) 4637.

[30] I.S. Jacobs, J.W. Bray, H.R. Hart Jr., L.V. Interrante, J.S. Kasper, G.D. Watkins, D.E. Prober and J.C. Bonner, Phys. Rev. B14 (1976) 3036.

[31] K.A. Penson, A. Holz and K.H. Benneman, in: Proc. of the Intern. Conf. on Magnetism ICM-76, III (Amsterdam, 1976) p. 1135.

[32] M.C. Cross and D.S. Fisher, Phys. Rev. B19 (1979) 402.

[33] P.W. Anderson, Mat. Res. Bull. 8 (1973) 153.

[34] P. Fazekas and P.W. Anderson, Phil. Mag. 30 (1974) 423.

[35] F. Stern, Phys. Rev. 94 (1954) 1412.

[36] R. Kubo, Revs. Modern Phys. 25 (1953) 344.

[37] J.H. van Vleck, The Theory of Electric and Magnetic Susceptibilities (Oxford University Press, 1932) Ch. 12.

[38] W.J. Caspers, Physics 1 (1964) 45.

[39] McGraw-Hill, Encyclopedia of Science and Technology (McGraw-Hill, Book Cy., 1966).

[40] D.J. Thouless, The Quantum Mechanics of Many-body Systems (Academic Press, 1964).

[41] L.I. Schiff, Quantum Mechanics (McGraw-Hill Book Cy., 1968) p. 250

[42] C.K. Majumdar and D.K. Ghosh, J. Math. Phys. 10 (1969) 1388, 1399.

[43] Th. Niemeijer, J. Math. Phys. 12 (1971) 1487.

[44] M. Tinkham, Group Theory and Quantum Mechanics (McGraw-Hill Book Cy., 1964) p. 143. 